\title{
Rank One Strange Attractors in Periodically Kicked Lorenz System with Time-Delay
}

\author{
Wenjie Yang, Yiping Lin, and Yunxian Dai \\ Department of Applied Mathematics, Kunming University of Science and Technology, Kunming, Yunnan 650500, China \\ Correspondence should be addressed to Yiping Lin; linyiping689@163.com
}

Received 22 November 2014; Accepted 20 January 2015

Academic Editor: Garyfalos Papashinopoulos

Copyright ( 2015 Wenjie Yang et al. This is an open access article distributed under the Creative Commons Attribution License, which permits unrestricted use, distribution, and reproduction in any medium, provided the original work is properly cited.

\begin{abstract}
Rank one strange attractor in periodically kicked Lorenz system with time-delay is investigated. Our discussion is based on the theory of rank one maps formulated by Wang and Young. First, we develop the rank one chaotic theory to delayed systems. It is shown that strange attractors occur when periodically kicked delayed system undergoes a generic Hopf bifurcation. Then we use the theory to the periodically kicked Lorenz system with delay, and derivation of conditions for Hopf bifurcation and rank one chaos along with the results of numerical simulations are presented.
\end{abstract}

\section{Introduction}

In the 1970s, chaos characterized by SRB measure was first introduced by Sinai, Ruelle, and Bowen [1-3]. Since then, many results on SRB measure, strange attractor, Lyapunov exponent, and their applications were obtained [4-6]. In 2001, Wang and Young gave simple conditions that guarantee, for strongly dissipative maps, the existence of strange attractors with a single direction of instability and certain controlled behaviors [7]. In 2008, Wang and Young accomplished a more comprehensive understanding of the complicated geometric and dynamical structures of a specific class of nonuniformly hyperbolic homoclinic tangles. For certain differential equations, through their well-defined computational process, the existence of the indicated phenomenon of rank one chaos was verified [8]. In 2009, Chen and Han studied the existence of rank one chaos in the neighborhood of a periodically kicked stable limit cycle close to a heteroclinic cycle of a planar equation [9]. In 2012, Fang studied the synchronization between rank one chaotic systems without and with delay using linear delayed feedback control method [10]. In 2013, Wang and Young gave a dynamical study of certain classes of rank one attractors [11].

The system we consider in this paper is the well-known Lorenz system with time-delay. Since Lorenz presented the Lorenz system as (1) in [12], people had considerable interest in the three-dimensional autonomous chaotic system (see, e.g., $[13,14])$ :

$$
\begin{aligned}
& \dot{x}=a(y-x), \\
& \dot{y}=c x-x z-y, \\
& \dot{z}=x y-b z .
\end{aligned}
$$

The following Lorenz system with time-delay has been researched [15]:

$$
\begin{aligned}
& \dot{x}(t)=a\left(y\left(t-\tau_{2}\right)-x\left(t-\tau_{1}\right)\right), \\
& \dot{y}(t)=c x\left(t-\tau_{1}\right)-x\left(t-\tau_{1}\right) z\left(t-\tau_{3}\right)-y\left(t-\tau_{2}\right), \\
& \dot{z}(t)=x\left(t-\tau_{1}\right) y\left(t-\tau_{2}\right)-b z\left(t-\tau_{3}\right) .
\end{aligned}
$$

The authors found that a simple time-delay transforms the Lorenz system to the generalized Chen system or the generalized Lü system without any coefficient changes.

In this paper, we consider the derivative of $x(t)$ with respect to $a(y(t)-x(t-\tau))$ and the derivative of $y(t)$ with 
respect to a time-periodic forcing term $\varepsilon P_{T}$. Then we consider the following system:

$$
\begin{aligned}
& \dot{x}(t)=a(y(t)-x(t-\tau)), \\
& \dot{y}(t)=c x(t)-x(t) z(t)-y(t)+\varepsilon P_{T} y(t), \\
& \dot{z}(t)=x(t) y(t)-b z(t),
\end{aligned}
$$

where $a, b$, and $c$ are real parameters. The stability of equilibrium, the bifurcating of periodic solutions, and the rank one chaos of the periodic kicked delayed system are investigated.

This paper is organized as follows. In Section 2, we give preliminaries about the rank one theory. In Section 3, we derived the rank one theory for delayed systems. In Section 4, we consider rank one chaos for the periodically kicked Lorenz system, and at last, numerical simulations are presented in Section 5.

\section{Preliminaries}

To properly motivate the studies presented in this paper, we first give a brief overview on the studies of rank one strange attractors, which can be constructed in the following way according to [16].

Let $\mathbf{u} \in R^{m}$, let $m \geq 2$ be the phase variable, and let $t \in R$ be the time. Consider the following system of equations:

$$
\frac{d \mathbf{u}}{d t}=\mathbf{A}_{\mu} \mathbf{u}+f_{\mu}(\mathbf{u})+\varepsilon P_{T} \Phi(\mathbf{u})
$$

where $\mathbf{A}_{\mu}$ is a real $m$ by $m$ matrix and $f_{\mu}(\mathbf{u})$ is a vector valued real analytic function in $\mathbf{u}$ defined on a given neighborhood of $\mathbf{u}=\mathbf{0}$ such that $f(\mathbf{0})=\mathbf{0}, D_{\mathbf{u}} f(\mathbf{0})=\mathbf{0}$. Both $\mathbf{A}_{\mu}$ and $f_{\mu}(\mathbf{u})$ are smooth dependents of parameter $\mu$ around $\mu=0$. $\varepsilon$ is a parameter that controls the magnitude of the forcing. $\Phi(\mathbf{u})$ is a real vector valued function of $\mathbf{u}$ that represents the shape of the forcing and $P_{T}=\sum_{n=-\infty}^{\infty} \delta(t-n T)$. When $\varepsilon=0$, the undisturbed system is

$$
\frac{d \mathbf{u}}{d t}=\mathbf{A}_{\mu} \mathbf{u}+f_{\mu}(\mathbf{u}) .
$$

Firstly, we give several definitions.

Definition 1 (see [17]). Let $f: M \rightarrow M$ be a diffeomorphism of a compact Riemannian manifold onto itself. One says $f$ is an Anosov diffeomorphism if the tangent space at every $x \in M$ is split into $E^{\mathcal{u}}(x) \oplus E^{s}(x)$ where $E^{u}$ and $E^{s}$ are $D f$-invariant subspaces, $\left.D f\right|_{E^{u}}$ is uniformly expanding, and $\left.D f\right|_{E^{s}}$ is uniformly contracting. A compact $f$-invariant set $\Lambda \subset M$ is called an attractor if there is a neighborhood $U$ of $\Lambda$ called its basin such that $f^{n} x \rightarrow \Lambda$ for every $x \in U . \Lambda$ is called an Axiom A attractor if the tangent bundle over $\Lambda$ is split into $E^{\mathcal{u}} \oplus E^{\mathcal{s}}$ as above.

Definition 2 (see [17]). Let $f$ be a $C^{2}$ diffeomorphism with an Axiom $A$ attractor $\Lambda$. Then there is a unique $f$-invariant Borel probability measure $\mu$ on $\Lambda$ that is characterized by each of the following (equivalent) conditions:

(i) $\mu$ has absolutely continuous conditional measures on unstable manifolds; (ii) consider

$$
h_{\mu}(f)=\int\left|\operatorname{det}\left(\left.D f\right|_{E^{u}}\right)\right| d \mu
$$

where $h_{\mu}(f)$ is the metric entropy of $f$;

(iii) there is a set $V \subset U$ having full Lebesgue measure such that, for every continuous observable $\varphi: U \rightarrow$ $R$, we have, for every $x \in V$,

$$
\frac{1}{n} \sum_{i=0}^{n-1} \varphi\left(f^{i} x\right) \longrightarrow \int \varphi d \mu
$$

(iv) $\mu$ is the zero-noise limit of small random perturbations of $f$.

Then the invariant measure $\mu$ is called the Sinai-RuelleBowen measure or SRB measure of $f$.

Definition 3. Given a map $f$, the Lyapunov exponent is defined at $x_{0}$ as follows:

$$
\begin{aligned}
\lambda\left(x_{0}\right) & =\lim _{N \rightarrow+\infty} \frac{1}{N} \ln \left|\prod_{i=0}^{N-1} f^{\prime}\left(x_{i}\right)\right| \\
& =\lim _{N \rightarrow+\infty} \frac{1}{N} \sum_{i=0}^{N-1} \ln \left|f^{\prime}\left(x_{i}\right)\right|,
\end{aligned}
$$

where $x_{i} \triangleq f\left(x_{i-1}\right)(i=1,2, \ldots, N-1)$.

For $f$ such that $f(\bar{U}) \subset U$ where $U \subset M$ is open, let $\Omega=\bigcap_{n \geq 0} f^{n}(\bar{U})$ be the attractor. By strange attractor, here we refer to attractors with the following three properties, see [18].

(SA1) Positive Lyapunov exponents: For Lebesgue, almost every $x \in U$; the orbit of $x$ has a positive Lyapunov exponent.

(SA2) Existence of SRB measure and basin property:

(a) $f$ admits at least one and at most finitely many ergodic SRB measures all of which have no zero Lyapunov exponents, denoted by $\mu_{1}, \mu_{2}, \ldots, \mu_{k}$.

(b) For Lebesgue, almost every $x \in U ; \exists j=j(x)$ such that, for every continuous function $\phi$ : $U \rightarrow R$,

$$
\frac{1}{n} \sum_{i=0}^{n-1} \phi\left(f^{i} x\right) \longrightarrow \int \phi d \mu_{j}
$$

(SA3) Statistical properties of dynamical observations:

(a) For every ergodic SRB measure $\mu$ and every Hölder continuous function $\phi: \Omega \rightarrow R$, the sequence $\left\{\phi \circ f^{i}\right\}_{i=0,1, \ldots}$ obeys a central limit theorem; that is, if $\int \phi d \mu=0$, then $1 / \sqrt{n} \sum_{i=0}^{n-1} \phi$ 。 
$f^{i}$ converges in distribution to the normal distribution, and the variance is strictly positive unless $\phi \circ f=\psi \circ f-\psi$ for some $\psi$.

(b) Suppose that, for some $N \geq 1, f^{N}$ has a SRB measure $\mu$ that is mixing. Then given Hölder exponent $\eta, \exists \tau=\tau(\eta)<1$ such that, for all $\varphi, \psi: \Omega \rightarrow R$ Hölder with exponent $\eta, \exists K=$ $K(\varphi, \psi)$ such that, for all $n \geq 1$,

$$
\left|\int\left(\varphi \circ f^{n}\right) \psi d \mu-\int \varphi d \mu \int \psi d \mu\right| \leq K(\varphi, \psi) \tau^{n} .
$$

Now, we consider $(2.1)_{\varepsilon}$. Assume the following.

(A1) Let $\left\{\lambda_{i}\right\}_{i=1}^{m}$ be the eigenvalues of $\mathbf{A}_{\mu}$. There is a conjugated pair

$$
\lambda_{1,2}=\alpha(\mu) \pm i \omega(\mu)
$$

such that $\alpha(0)=0, \omega(0) \neq 0,(d / d \mu) \alpha(0) \neq 0$, and there exists $c>0$ such that $\operatorname{Re}\left(\lambda_{i}\right)<-c, i \geq 3$.

Then the flow on the central manifold $W^{c}$ of system $(2.1)_{0}$ can be explicitly written by using a complex variable $z$ in the following normal form:

$$
\begin{aligned}
\frac{d z}{d t}= & (\alpha(\mu)+i \omega(\mu)) z+g_{20}(\mu) \frac{z^{2}}{2}+g_{11}(\mu) z \bar{z} \\
& +g_{02}(\mu) \frac{\bar{z}^{2}}{2}+g_{21}(\mu) \frac{z^{2} \bar{z}}{2}+\cdots
\end{aligned}
$$

According to the standard normal form theory, there exists a change of variables near identity, which we write as

$$
z=Z+\Psi(Z, \bar{Z})=Z+\sum_{2 \leq j+k \leq L} \psi_{j k} \frac{Z^{j} \bar{Z}^{k}}{j ! k !},
$$

which transfers (10) to

$$
\frac{d Z}{d t}=(\alpha(\mu)+i \omega(\mu)) Z+k_{1}(\mu) Z^{2} \bar{Z}+\cdots
$$

Using the formula given in [19], we have

$$
k_{1}(0)=\frac{i}{2 \omega}\left(g_{20} g_{11}-2\left|g_{11}\right|^{2}-\frac{1}{3}\left|g_{02}\right|^{2}\right)+\frac{g_{21}}{2} .
$$

Assume the following.

(A2) $\operatorname{Re}\left(k_{1}(0)\right)<0$. Suppose that a real linear coordinate change $\mathbf{u} \rightarrow L_{\mu} \mathbf{u}:=(\xi, \eta, \mathbf{w})^{T}$ transfers $(2.1)_{\varepsilon}$ into

$$
\begin{aligned}
& \frac{d \xi}{d t}=\alpha \xi+\omega \eta+f_{\xi}+\varepsilon \Phi_{\xi} P_{T}(t), \\
& \frac{d \eta}{d t}=-\omega \xi+\alpha \eta+f_{\eta}+\varepsilon \Phi_{\eta} P_{T}(t), \\
& \frac{d \mathbf{w}}{d t}=A^{(s)} \mathbf{w}+\omega \eta+f_{\mathbf{w}}+\varepsilon \Phi_{\mathbf{w}} P_{T}(t),
\end{aligned}
$$

where $\xi, \eta$ are scalars. w is an $m-2$-vector, $A^{(s)}$ is an $(m-2)$ by $(m-2)$ matrix of stable eigenvalues, and

$$
\begin{gathered}
\left(f_{\xi}, f_{\eta}, f_{\mathbf{w}}\right)^{T}=L_{\mu} f_{\mu}\left(L_{\mu}^{-1}(\xi, \eta, \mathbf{w})^{T}\right), \\
\left(\Phi_{\xi}, \Phi_{\eta}, \Phi_{\mathbf{w}}\right)^{T}=L_{\mu} \Phi\left(L_{\mu}^{-1}(\xi, \eta, \mathbf{w})^{T}\right) .
\end{gathered}
$$

Let $(\widehat{r}, \widehat{\mathbf{s}}) \in R^{+} \times S^{m-1}=\widehat{r} \widehat{\mathbf{s}}$ be such that $(\xi, \eta, \mathbf{w})^{T}=\widehat{r} \widehat{\mathbf{s}}$, and define

$$
\left(\Psi_{\xi}(\widehat{r}, \widehat{\mathbf{s}}), \Psi_{\eta}(\widehat{r}, \widehat{\mathbf{s}}), \Psi_{w}(\widehat{r}, \widehat{\mathbf{s}})\right)^{T}=\left(\Phi_{\xi}, \Phi_{\eta}, \Phi_{\mathbf{w}}\right)^{T} .
$$

Further let $\left\{\widehat{\mathbf{s}}_{0}=(\cos \theta, \sin \theta, \mathbf{0}) \in S^{m-1}, \theta \in[0,2 \pi)\right\}$ be the unit circle in $(\xi, \eta)$-plane in $(\xi, \eta, \mathbf{w})$-space, and define

$$
\varphi(\theta)=\cos \theta \Psi_{\xi}\left(0, \widehat{\mathbf{s}}_{0}\right)+\sin \theta \Psi_{\eta}\left(0, \widehat{\mathbf{s}}_{0}\right) .
$$

The time- $T$ map of $(2.1)_{\varepsilon}$ is denoted by $F_{\mu, \varepsilon, T}$, where $\mu$ is the bifurcation parameter of the unperturbed $(2.1)_{0}$ and $\varepsilon, T$ are the parameters of forcing. Assume that
(a) (A1)-(A2) hold for $(2.1)_{\mathcal{\varepsilon}}$;
(b) $\varphi(\theta)$ in (13) is a Morse function;
(c) $\mu$, $\varepsilon$ are such that $0<\mu \ll 1$ and $0<\varepsilon \ll 1$.

The following Theorem is obtained by Wang and Oksasoglu [16].

Theorem 4. Let the values of $\mu$ and $\varepsilon$ be fixed and assume that (a) -(c) hold. Regard period $T$ of the forcing as a parameter and denote $F_{T}=F_{\mu, \varepsilon, T}$. Then there exists a constant $K_{1}$, determined exclusively by $\varphi(\theta)$, such that if

$$
\left|\varepsilon \frac{\operatorname{Im}\left(k_{1}(0)\right)}{\operatorname{Re}\left(k_{1}(0)\right)}\right|>K_{1},
$$

then there exists a positive measure set $\Delta \subset\left(\mu^{-1}, \infty\right)$ for $T$, so that, for $T \in \Delta, F_{T}$ has a strange attractor $\Lambda$ admitting no periodic sinks. That is to say, there exists an open neighborhood $U$ of $\Lambda$ in $\mathbb{R}^{m}$ such that $F_{T}$ has a positive Lyapunov exponent for Lebesgue almost every point in $U$. Furthermore, $F_{T}$ admits an ergodic SRB measure, with respect to which almost every point of $U$ is generic.

\section{Rank One Strange Attractors of Delayed System}

In this section we consider a nonlinear delayed differential equation:

$$
\frac{d \mathbf{u}}{d t}=L_{\mu} \mathbf{u}_{t}+f_{\mu}\left(\mathbf{u}_{t}\right)+\varepsilon P_{T} \Phi\left(\mathbf{u}_{t}\right)
$$

where $\mathbf{u}_{t}(\theta)=\mathbf{u}(t+\theta), \theta \in[-\tau, 0]$ for $\tau>0, L_{\mu}$ : $C[-\tau, 0] \rightarrow R^{n}$ is a linear operator, and $f_{\mu}: C[-\tau, 0] \rightarrow R^{n}$ is a nonlinear term satisfying $f_{\mu}(\mathbf{0})=\mathbf{0}, D_{\mathbf{u}} f_{\mu}(\mathbf{0})=\mathbf{0}$. $f_{\mu}$ and $L_{\mu}$ depend on $\mu$ analytically for $|\mu|$ is sufficiently small, and 
$P_{T}=\sum_{n=-\infty}^{\infty} \delta(t-n T)$. When $\varepsilon=0$, the undisturbed system is

$$
\frac{d \mathbf{u}}{d t}=L_{\mu} \mathbf{u}_{t}+f_{\mu}\left(\mathbf{u}_{t}\right)
$$

For linear system $\dot{\mathbf{u}}=L_{\mu} \mathbf{u}_{t}$, there is an $n \times n$ matrix $\eta(\cdot, \mu)$ : $[-\tau, 0] \rightarrow R^{n^{2}}$, such that for any $\phi \in C[-\tau, 0]$

$$
L_{\mu} \phi=\int_{-\tau}^{0} d \eta(\theta, \mu) \phi(\theta)
$$

Let the spectral set of $L_{\mu}$

$$
\sigma(\mu)=\left\{\lambda \mid \operatorname{det}\left(\lambda I-L_{\mu} e^{\lambda \theta} I\right)=0\right\}
$$

satisfy the following.

(B1) There is a simple conjugated pair

$$
\lambda_{1,2}=a(\mu) \pm i \omega(\mu)
$$

such that $a(0)=0, \omega(0)=\omega_{0}>0,(d / d \mu) a(0) \neq 0$, and there exists $c>0$ such that, for any $\lambda \in \sigma(\mu), \lambda \neq \lambda_{1,2} ; \operatorname{Re}\left(\lambda_{i}\right)<-c$, $i \geq 3$.

Then the flow on the central manifold $W^{c}$ of system $(3.1)_{0}$ can be written by using Hassard's method [20] in the following form:

$$
\begin{aligned}
\dot{z} & =i \omega_{0} z+g(z, \bar{z}) \\
& =i \omega_{0} z+\frac{g_{20}}{2} z^{2}+g_{11} z \bar{z}+\frac{g_{02}}{2} \bar{z}^{2}+\frac{g_{21}}{2} z^{2} \bar{z}+\cdots,
\end{aligned}
$$

at $\mu=0$. Let

$$
k_{1}(0)=\frac{i}{2 \omega_{0}}\left(g_{20} g_{11}-2\left|g_{11}\right|^{2}-\frac{1}{3}\left|g_{02}\right|^{2}\right)+\frac{g_{21}}{2} .
$$

\section{Suppose the following.}

(B2) $\operatorname{Re}\left(k_{1}(0)\right)<0$. Then we know that system $(3.1)_{0}$ has a supercritical Hopf bifurcation near the equilibrium.

We define for $\phi \in C[-\tau, 0]$

$$
\begin{aligned}
A(\mu) \phi & = \begin{cases}\frac{d \phi}{d \theta}, & \theta \in[-\tau, 0), \\
\int_{-\tau}^{0} d \eta(s, \mu) \phi(s)=L_{\mu} \phi, & \theta=0,\end{cases} \\
R \phi & = \begin{cases}0, & \theta \in[-\tau, 0), \\
f_{\mu}(\phi)+\varepsilon P_{T} \Phi(\phi), & \theta=0 .\end{cases}
\end{aligned}
$$

Since $d \mathbf{u}_{t} / d \theta=d \mathbf{u}_{t} / d t,(3.1)_{\varepsilon}$ becomes

$$
\dot{\mathbf{u}}_{t}=A(\mu) \mathbf{u}_{t}+R \mathbf{u}_{t} \text {. }
$$

For $\theta=0(25)$ is $(3.1)_{\varepsilon}$.

Following Hassard's method, we know that $z$ satisfies equation

$$
z(t)=\left\langle q^{*}(\theta), u_{t}(\theta)\right\rangle
$$

where $q^{*}(\theta)$ is a eigenvector corresponding to eigenvalue $-i \omega_{0}$ of $A^{*}$ which is the adjoint operator of $A(0)$.

When $\mu=0$, let $A=A(0)$ and we define

$$
\begin{aligned}
W(t, \theta) & =u_{t}(\theta)-z(t) q(\theta)-\bar{z}(t) \bar{q}(\theta) \\
& =u_{t}(\theta)-2 \operatorname{Re}\{z(t) q(\theta)\} \\
& =W(z(t), \bar{z}(t), \theta) .
\end{aligned}
$$

Then from $(3.1)_{\varepsilon}$ and the definitions of $A, A^{*}$, we obtain

$$
\begin{aligned}
\dot{z} & =\left\langle q^{*}(\theta), \dot{u}_{t}(\theta)\right\rangle=\left\langle q^{*}(\theta), A u_{t}(\theta)+R u_{t}(\theta)\right\rangle \\
& =\left\langle q^{*}(\theta), A u_{t}(\theta)\right\rangle+\left\langle q^{*}(\theta), R u_{t}(\theta)\right\rangle \\
& =\left\langle A^{*} q^{*}(\theta), u_{t}(\theta)\right\rangle+\left\langle q^{*}(\theta), R u_{t}(\theta)\right\rangle \\
& =i \omega_{0}\left\langle q^{*}(\theta), u_{t}(\theta)\right\rangle+\left\langle q^{*}(\theta), R u_{t}(\theta)\right\rangle .
\end{aligned}
$$

At $\theta=0$,

$$
\begin{aligned}
\dot{z}= & i \omega_{0} z+\overline{q^{*}}(0) f_{0}(W(z, \bar{z}, 0)+2 \operatorname{Re} z q(0)) \\
& +\varepsilon P_{T} \overline{q^{*}}(0) \Phi(W(z, \bar{z}, 0)+2 \operatorname{Re} z q(0)) \\
= & i \omega_{0} z+g(z, \bar{z}) \\
& +\varepsilon P_{T} \overline{q^{*}}(0) \Phi(W(z, \bar{z}, 0)+2 \operatorname{Re} z q(0)) .
\end{aligned}
$$

We can calculate

$$
\begin{aligned}
\dot{W}= & \dot{u}_{t}-\dot{z} q-\dot{\bar{z}} \bar{q}=A W+H(z, \bar{z}, 0) \\
= & \frac{H_{20}(\theta)}{2} z^{2}+H_{11}(\theta) z \bar{z}+\frac{H_{02}(\theta)}{2} \bar{z}^{2} \\
& +\frac{H_{30}(\theta)}{6} z^{3}+\cdots
\end{aligned}
$$

Then (25) can be written as

$$
\begin{aligned}
\dot{z}= & i \omega_{0} z+\frac{g_{20}}{2} z^{2}+g_{11} z \bar{z}+\frac{g_{02}}{2} \bar{z}^{2}+\frac{g_{21}}{2} z^{2} \bar{z}+\cdots \\
& +\varepsilon P_{T} \overline{q^{*}}(0) \Phi(W(z, \bar{z}, 0)+2 \operatorname{Re} z q(0)), \\
\dot{W}= & A W+H(z, \bar{z}, 0) .
\end{aligned}
$$

Let $W \in \mathfrak{B}$, where $\mathfrak{B}$ is a Banach space, and let $z=x+i y$ in (31). Define

$$
\begin{aligned}
& \Psi_{x}(x, y) \\
& \quad=\operatorname{Re}\left\{\overline{q^{*}}(0) \Phi(W(x, y, 0)+2 \operatorname{Re}(x+i y) q(0))\right\}, \\
& \Psi_{y}(x, y) \\
& \quad=\operatorname{Im}\left\{\overline{q^{*}}(0) \Phi(W(x, y, 0)+2 \operatorname{Re}(x+i y) q(0))\right\} .
\end{aligned}
$$

We further let $x=\cos \theta, y=\sin \theta$, and $\mathbf{W}=\mathbf{0}$ in (32); then $\left\{\widehat{\mathbf{s}}_{0}=(\cos \theta, \sin \theta, \mathbf{0}) \in S \times \mathfrak{B}, \theta \in[0,2 \pi)\right\}$ is the unit circle in $(x, y)$-plane in $(x, y, \mathbf{W})$-space. Define

$$
\varphi(\theta)=\cos \theta \Psi_{x}\left(\widehat{\mathbf{s}}_{\mathbf{0}}\right)+\sin \theta \Psi_{y}\left(\widehat{\mathbf{s}}_{\mathbf{0}}\right) .
$$


The time- $T$ map of $(3.1)_{\varepsilon}$ is denoted by $F_{\mu, \varepsilon, T}$ where $\mu$ is the bifurcation parameter of the unperturbed $(3.1)_{0}$ and $\varepsilon, T$ are the parameters of forcing. Assume that

(a) (B1)-(B2) hold for $(3.1)_{\varepsilon}$;

(b) $\phi(\theta)$ in (33) is a Morse function;

(c) $\mu$, $\varepsilon$ are such that $0<\mu \ll 1,0<\varepsilon \ll 1$.

Then we obtain the following.

Theorem 5. Let the values of $\mu$ and $\varepsilon$ be fixed and assume that (a)-(c) hold. Regard period $T$ of the forcing as a parameter and denote $F_{T}=F_{\mu, \varepsilon, T}$. Then there exists a constant $K_{2}$, determined exclusively by $\varphi(\theta)$, such that if

$$
\left|\varepsilon \frac{\operatorname{Im}\left(k_{1}(0)\right)}{\operatorname{Re}\left(k_{1}(0)\right)}\right|>K_{2},
$$

then there exists a positive measure set $\Delta \subset\left(\mu^{-1}, \infty\right)$ for $T$, so that, for $T \in \Delta, F_{T}$ has a strange attractor $\Lambda$ admitting no periodic sinks. That is to say, there exists an open neighborhood $U$ of $\Lambda$ such that $F_{T}$ has a positive Lyapunov exponent for Lebesgue almost every point in $U$. Furthermore, $F_{T}$ admits an ergodic SRB measure, with respect to which almost every point of $U$ is generic.

Proof. We can easy see that (B1) and (B2) in Theorem 5 are corresponding to (A1) and (A2) in Theorem 4. After transformations, $(33)$ in $(x, y)$-plane is corresponding to (17) in $(\xi, \eta)$-plane, and they are on the central manifolds. Obviously the conditions in Theorem 4 are satisfied.

\section{Analysis of Rank One Strange Attractors in Delayed Lorenz System}

We consider the corresponding undisturbed system of (3) as follows:

$$
\begin{aligned}
& \dot{x}(t)=a(y(t)-x(t-\tau)), \\
& \dot{y}(t)=c x(t)-x(t) z(t)-y(t), \\
& \dot{z}(t)=x(t) y(t)-b z(t) .
\end{aligned}
$$

Obviously, system (35) always has equilibria $E_{i}^{*}\left(x_{i}^{*}, y_{i}^{*}, z_{i}^{*}\right)$, $i=1,2,3$, where

$$
\begin{aligned}
& x_{1}^{*}=0, \\
& y_{1}^{*}=0, \\
& z_{1}^{*}=0, \\
& x_{2}^{*}=y_{2}^{*}, \\
& y_{2}^{*}=\sqrt{b z_{2}^{*}},
\end{aligned}
$$

$$
\begin{aligned}
& z_{2}^{*}=c-1, \\
& x_{3}^{*}=y_{3}^{*}, \\
& y_{3}^{*}=-\sqrt{b z_{3}^{*},} \\
& z_{3}^{*}=c-1 .
\end{aligned}
$$

Let $E^{*}=\left(x^{*}, y^{*}, z^{*}\right)$ be the arbitrary equilibrium, and denote $u=\left(x_{1}(t), y_{1}(t), z_{1}(t)\right)^{T}$, where $x_{1}(t)=x(t)-x^{*}$, $y_{1}(t)=y(t)-y^{*}$, and $z_{1}(t)=z(t)-z^{*}$ still denote $x_{1}(t)$, $y_{1}(t)$, and $z_{1}(t)$ by $x(t), y(t)$, and $z(t)$, respectively; then the linearized system of the corresponding equation at $E^{*}$ is as follows:

$$
\dot{u}=A u(t)+B u(t-\tau),
$$

where $A=\left(a_{i j}\right)_{3 \times 3}, B=\left(b_{i j}\right)_{3 \times 3}, a_{12}=a, a_{21}=c-z^{*}, a_{22}=-1$, $a_{23}=-x^{*}, a_{31}=y^{*}, a_{32}=x^{*}, a_{33}=-b, b_{11}=-a$, and all the others of $a_{i j}$ and $b_{i j}$ are 0 .

The characteristic equation of system (37) is

$$
\lambda^{3}+\alpha_{1} \lambda^{2}+\alpha_{2} \lambda+\alpha_{3}+a e^{-\lambda \tau}\left(\lambda^{2}+\beta_{1} \lambda+\beta_{2}\right)=0
$$

where $\alpha_{1}=1+b, \alpha_{2}=b+x^{* 2}-a\left(c-z^{*}\right), \alpha_{3}=a x^{* 2}-a b\left(c-z^{*}\right)$, $\beta_{1}=1+b$, and $\beta_{2}=b+x^{* 2}$. We consider the following cases.

(1) One has $E^{*}=E_{1}^{*}$. The coefficients of (38) are $\alpha_{1}=1+b$, $\alpha_{2}=b-a c, \alpha_{3}=-a b c, \beta_{1}=\alpha_{1}$, and $\beta_{2}=b$.

When $\tau=0$, (38) becomes

$$
\begin{aligned}
\Gamma(\lambda) & =\lambda^{3}+\left(a+\alpha_{1}\right) \lambda^{2}+\left(\alpha_{2}+a \beta_{1}\right) \lambda+\alpha_{3}+a \beta_{2} \\
& =0 .
\end{aligned}
$$

Routh-Hurwitz criterion implies that if

$$
\begin{array}{r}
a+b+1>0, \\
b[a(a+b+3)+c(1+b-a)]>0, \\
2 a b^{2}(c-1)[a(a+b+3)+c(1+b-a)]>0,
\end{array}
$$

then (38) has at least one positive real root, so when $\left(H_{1}\right)$ holds, $E_{1}^{*}$ is unstable equilibrium.

(2) One has $E^{*}=E_{2}^{*}$, or $E^{*}=E_{3}^{*}$. Due to the symmetry of $E_{2}^{*}$ and $E_{3}^{*}$, here we only consider $E^{*}=E_{2}^{*}$. When $\tau=0$, the coefficients of system (38) are $\alpha_{1}=1+b, \alpha_{2}=b c-a$, $\alpha_{3}=a b(c-2), \beta_{1}=\alpha_{1}$, and $\beta_{2}=b c$. According to RouthHurwitz criterion, if $\left(H_{1}\right)$ holds, then all roots of (38) have negative real parts.

Suppose that $i \omega(\omega>0)$ is a root of (38) in the imaginary axis. Substituting it in (38) and separating the real and imaginary parts, we have

$$
\begin{aligned}
& a \beta_{1} \omega \cos (\omega \tau)-a\left(\beta_{2}-\omega^{2}\right) \sin (\omega \tau)=\omega^{3}-\alpha_{2} \omega, \\
& a \beta_{1} \omega \sin (\omega \tau)+a\left(\beta_{2}-\omega^{2}\right) \cos (\omega \tau)=\alpha_{1} \omega^{2}-\alpha_{3},
\end{aligned}
$$


which is equivalent to

$$
\omega^{6}+e_{1} \omega^{4}+e_{2} \omega^{2}+e_{3}=0 .
$$

Let $z=\omega^{2}$; then (41) becomes

$$
G(z) \triangleq z^{3}+e_{1} z^{2}+e_{2} z+e_{3}=0,
$$

where $e_{1}=\alpha_{1}^{2}-2 \alpha_{2}-a^{2}, e_{2}=2 a^{2} \beta_{2}+\alpha_{2}^{2}-a^{2} \beta_{1}^{2}-2 \alpha_{1} \alpha_{3}$, and $e_{3}=\alpha_{3}^{2}-a^{2} \beta_{2}^{2}$.

Lemma 6. For (42), one has the following.

(i) If $e_{3}<0$, then (42) has at least one positive root.

(ii) If $e_{3} \geqslant 0$, then (42) has at least one positive root if and only if there exists $z^{*}>0$ such that $G^{\prime}\left(z^{*}\right)=0$ and $G\left(z^{*}\right) \leqslant 0$.

We assume that (42) has three positive roots $z_{1}, z_{2}$, and $z_{3}$ and $\omega_{1}=\sqrt{z_{1}}, \omega_{2}=\sqrt{z_{2}}$, and $\omega_{3}=\sqrt{z_{3}}$.

According to (40), we have

$$
\tau_{k}^{(j)}=\frac{1}{\omega_{k}}\left\{\arcsin \frac{\omega_{k}^{5}+\left(\alpha_{1} \beta_{1}-\alpha_{2}-\beta_{2}\right) \omega_{k}^{3}+\left(\alpha_{2} \beta_{2}-\alpha_{3} \beta_{1}\right) \omega_{k}}{a \beta_{1}^{2} \omega_{k}^{2}+a\left(\beta_{2}-\omega_{k}^{2}\right)^{2}}+2 j \pi\right\},
$$

where $k=1,2,3$ and $j=0,1, \ldots$; then $\pm i \omega_{k}$ is a pair of imaginary roots of (38) with $\tau_{k}^{(j)}$.

Define

$$
\begin{aligned}
& \tau_{0}=\tau_{k_{0}}^{(0)}=\min _{k=1,2,3}\left\{\tau_{k}^{(0)}\right\}, \\
& \omega_{0}=\omega_{k_{0}} .
\end{aligned}
$$

In order to investigate the distribution of the roots of (38), we need to introduce the following lemmas [21].

Lemma 7. Consider the exponential polynomial

$$
\begin{aligned}
P( & \left.\lambda, e^{-\lambda \tau_{1}}, \ldots, e^{-\lambda \tau_{m}}\right) \\
= & \lambda^{n}+p_{1}^{(0)} \lambda^{n-1}+\cdots+p_{n-1}^{(0)} \lambda+p_{n}^{(0)} \\
= & {\left[p_{1}^{(1)} \lambda^{n-1}+\cdots+p_{n-1}^{(1)} \lambda+p_{n}^{(1)}\right] e^{-\lambda \tau_{1}}+\cdots } \\
& +\left[p_{1}^{(m)} \lambda^{n-1}+\cdots+p_{n-1}^{(m)} \lambda+p_{n}^{(m)}\right] e^{-\lambda \tau_{m}},
\end{aligned}
$$

where $\tau_{i} \geq 0, i=1,2, \ldots, m$, and $p_{j}^{(i)}(i=0,1, \ldots, m ; j=$ $1,2, \ldots, n)$ are constants. As $\left(\tau_{1}, \tau_{2}, \ldots, \tau_{m}\right)$ vary, the sum of the order of the zeros of $P\left(\lambda, e^{-\lambda \tau_{1}}, \ldots, e^{-\lambda \tau_{m}}\right)$ on the open right half plane can change if and only if there are zeros crossing the imaginary axis.

Let $\lambda(\tau)=\alpha(\tau)+i \omega(\tau)$ be the root of (38) near $\tau=\tau_{k}^{(j)}$ satisfying

$$
\begin{aligned}
& \alpha\left(\tau_{k}^{(j)}\right)=0, \\
& \omega\left(\tau_{k}^{(j)}\right)=\omega_{k} .
\end{aligned}
$$

Then, the following transversal condition holds.
Lemma 8. Suppose that $z_{k}=\omega_{k}^{2}$ and $G^{\prime}\left(z_{k}\right) \neq 0$; then $\operatorname{Re} \lambda\left(\tau_{k}^{(j)}\right) / d \tau$ has the same sign as $G^{\prime}\left(z_{k}\right)$.

Proof. Substituting $\lambda(\tau)$ into (38) and taking the derivative with respect to $\tau$, we obtain

$$
\begin{aligned}
& \left\{3 \lambda^{2}+2 \alpha_{1} \lambda+\alpha_{2}\right. \\
& \left.\quad+a e^{-\lambda \tau}\left[2 \lambda+\beta_{1}-\tau\left(\lambda^{2}+\beta_{1} \lambda+\beta_{2}\right)\right]\right\} \frac{d \lambda}{d \tau} \\
& =a \lambda e^{-\lambda \tau}\left(\lambda^{2}+\beta_{1} \lambda+\beta_{2}\right) .
\end{aligned}
$$

Therefore,

$$
\begin{aligned}
{\left[\frac{d \lambda}{d \tau}\right]^{-1}=} & \frac{\left(3 \lambda^{2}+2 \alpha_{1} \lambda+\alpha_{2}\right) e^{\lambda \tau}}{a \lambda\left(\lambda^{2}+\beta_{1} \lambda+\beta_{2}\right)} \\
& +\frac{2 a \lambda+a \beta_{1}}{a \lambda\left(\lambda^{2}+\beta_{1} \lambda+\beta_{2}\right)}-\frac{\tau}{\lambda} .
\end{aligned}
$$

When $\tau=\tau_{k}^{(j)}, \lambda=i \omega_{k}, k=1,2$, we have

$$
\begin{aligned}
& {\left[\left(3 \lambda^{2}+2 \alpha_{1} \lambda+\alpha_{2}\right) e^{\lambda \tau}\right]_{\tau=\tau_{k}^{(j)}}} \\
& =\left(\left(\alpha_{2}-3 \omega_{k}^{2}\right) \cos \left(\omega_{k} \tau_{k}^{(j)}\right)-2 \alpha_{1} \omega_{k} \sin \left(\omega_{k} \tau_{k}^{(j)}\right)\right) \\
& +\left(2 \alpha_{1} \omega_{k} \cos \left(\omega_{k} \tau_{k}^{(j)}\right)+\left(\alpha_{2}-3 \omega_{k}^{2}\right) \sin \left(\omega_{k} \tau_{k}^{(j)}\right)\right) \\
& \quad \cdot i \\
& {\left[\lambda\left(\lambda^{2}+\beta_{1} \lambda+\beta_{2}\right)\right]_{\tau=\tau_{k}^{(j)}}=-\beta_{1} \omega_{k}^{2}+\omega_{k}\left(\beta_{2}-\omega_{k}^{2}\right) i,} \\
& {\left[2 a \lambda+a \beta_{1}\right]_{\tau=\tau_{k}^{(j)}}=a \beta_{1}+2 a \omega_{k} i .}
\end{aligned}
$$

According to (48), (49), and (42), we have

$$
\begin{aligned}
& {\left[\frac{d \operatorname{Re}(\lambda)}{d \tau}\right]_{\tau=\tau_{k}^{(j)}}^{-1}=\operatorname{Re}\left[\frac{\left(3 \lambda^{2}+2 \alpha_{1} \lambda+\alpha_{2}\right) e^{\lambda \tau}}{a \lambda\left(\lambda^{2}+\beta_{1} \lambda+\beta_{2}\right)}\right]_{\tau=\tau_{k}^{(j)}}} \\
& \quad+\operatorname{Re}\left[\frac{2 \lambda+\beta_{1}}{\lambda\left(\lambda^{2}+\beta_{1} \lambda+\beta_{2}\right)}\right]_{\tau=\tau_{k}^{(j)}} \\
& \quad=\frac{1}{\Lambda}\left\{-\left[2 a \omega_{k}^{4}+\omega_{k}^{2}\left(2 a \beta_{2}-a \beta_{1}\right)\right]\right. \\
& \quad+\left[\omega_{k}^{4}\left(3 \beta_{1}-2 \alpha_{1}\right)+\omega_{k}^{2}\left(2 \alpha_{1} \beta_{2}-\alpha_{2} \beta_{1}\right)\right] \\
& \quad \cdot \cos \left(\omega_{k} \tau_{k}^{(j)}\right) \\
& +\left[3 \omega_{k}^{5}+\omega_{k}^{3}\left(2 \alpha_{1} \beta_{1}-\alpha_{2}-3 \beta_{2}\right)+\alpha_{2} \beta_{2} \omega_{k}\right] \\
& \left.\quad \cdot \sin \left(\omega_{k} \tau_{k}^{(j)}\right)\right\}=\frac{1}{\Lambda}\left\{3 \omega_{k}^{6}+2\left(\alpha_{1}^{2}-2 \alpha_{2}-a^{2}\right) \omega_{k}^{4}\right.
\end{aligned}
$$




$$
\begin{aligned}
& \left.+\left(2 a^{2} \beta_{2}+\alpha_{2}^{2}-a^{2} \beta_{1}^{2}\right) \omega_{k}^{2}\right\} \\
& =\left[\frac{z_{k}\left(3 z_{k}^{2}+2 e_{1} z_{k}+e_{2}\right)}{\Lambda}\right]_{\tau=\tau_{k}^{(j)}}^{-1}=\frac{z_{k} G^{\prime}\left(z_{k}\right)}{\Lambda},
\end{aligned}
$$

where $\Lambda=a^{2} \beta_{1}^{2} \omega_{k}^{4}+a^{2} \omega_{k}^{2}\left(\beta_{2}-\omega_{k}^{2}\right)^{2}>0$.

Therefore, $\operatorname{Re} \lambda\left(\tau_{k}^{(j)}\right) / d \tau$ has the same sign as $G^{\prime}\left(z_{k}\right)$.

Now we apply the Hopf bifurcation theorem for functional differential equations [22] and get the following results.

Theorem 9. Suppose that $\left(H_{1}\right)$ and Lemma 6 are satisfied. Then

(i) when $\tau \in\left[0, \tau_{0}\right)$, equilibrium $E_{2}^{*}$ of system (35) is locally asymptotically stable;

(ii) when $\tau>\tau_{0}$, equilibrium $E_{2}^{*}$ of system (35) is unstable;

(iii) when $\tau=\tau_{0}$, then system (35) undergoes Hopf bifurcation at equilibrium $E_{2}^{*}$.

Now, we will derive the explicit formulae determining the direction and stability of these periodic solutions bifurcating from equilibrium $E_{2}^{*}$. Let $\tau=\tau_{0}+\mu, t=\tau \bar{t}$, and omit "-" above $t$; we rewrite (3) as

$$
\begin{gathered}
\dot{u}(t)=L_{\mu} u_{t}+f\left(\mu, u_{t}\right)+\varepsilon P_{T} \Phi(\phi), \\
L_{\mu}(\phi)=\left(\tau_{0}+\mu\right) A\left[\begin{array}{l}
\phi_{1}(0) \\
\phi_{2}(0) \\
\phi_{3}(0)
\end{array}\right] \\
+\left(\tau_{0}+\mu\right) B\left[\begin{array}{l}
\phi_{1}(-1) \\
\phi_{2}(-1) \\
\phi_{3}(-1)
\end{array}\right], \\
f(\mu, \phi)=\left(\tau_{0}+\mu\right)\left[\begin{array}{c}
0 \\
-\phi_{1}(0) \phi_{3}(0) \\
\phi_{1}(0) \phi_{2}(0)
\end{array}\right], \\
\Phi(\phi)=\left(\tau_{0}+\mu\right)\left[\begin{array}{c}
0 \\
\phi_{2}(0) \\
0
\end{array}\right],
\end{gathered}
$$

where $A, B$ are as in (37). We write $L_{\mu} \phi$ as the form $L_{\mu} \phi=$ $\int_{-1}^{0} d \eta(\theta, \mu) \phi(\mu)$, where

$$
d \eta(\theta, \mu)=\left(\tau_{0}+\mu\right) A \delta(\theta)+\left(\tau_{0}+\mu\right) B \delta(\theta+1),
$$

where $\delta(\theta)$ is the Dirac delta function. Then (3) can be written as

$$
\dot{u}_{t}=A(\mu) u_{t}+R u_{t}
$$

where

$$
\begin{aligned}
A(\mu) \phi & = \begin{cases}\frac{d \phi(\theta)}{d \theta}, & \theta \in[-1,0), \\
\int_{-1}^{0} d \eta(s, \mu) \phi(s), & \theta=0,\end{cases} \\
R \phi & = \begin{cases}0, & \theta \in[-1,0), \\
f_{\mu}(\phi)+\varepsilon P_{T} \Phi(\phi), & \theta=0 .\end{cases}
\end{aligned}
$$

Assume that $q(\theta)$ is the eigenvector of $A(0)$ corresponding to $i \omega_{0} \tau_{0}$; then $A(0) q(\theta)=i \omega_{0} \tau_{0} q(\theta)$. It follows from the definition of $A(0)$ that

$$
\left(\begin{array}{ccc}
i \omega_{0}+a e^{-i \omega_{0} \tau_{0}} & -a & 0 \\
-1 & i \omega_{0}+1 & x^{*} \\
-x^{*} & -x^{*} & i \omega_{0}+b
\end{array}\right) q(\theta)=\left(\begin{array}{l}
0 \\
0 \\
0
\end{array}\right) .
$$
where

Thus, we can easily compute $q(\theta)=(1, \alpha, \beta)^{T} e^{i \omega_{0} \tau_{0} \theta}$,

$$
\begin{aligned}
& \alpha=\frac{a e^{-i \omega_{0} \tau_{0}}+i \omega_{0}}{a}, \\
& \beta=\frac{x^{*}(1+\alpha)}{b+i \omega_{0}} .
\end{aligned}
$$

Similarly, it can be verified that $q^{*}(s)=D\left(1, \alpha^{*}, \beta^{*}\right) e^{i \omega_{0} \tau_{0}}$ is the eigenvector of $A^{*}$ corresponding to $-i \omega_{0} \tau_{0}$, where

$$
\begin{aligned}
& \alpha^{*}=\frac{\left(a e^{i \omega_{0} \tau_{0}}-i \omega_{0}\right)\left(i \omega_{0}-b\right)}{x^{* 2}-b+i \omega_{0}}, \\
& \beta^{*}=\frac{x^{*} \alpha^{*}}{i \omega_{0}-b} .
\end{aligned}
$$

In order to assure the bilinear inner product $\left\langle q^{*}(s), q(\theta)\right\rangle=1$, we have

$$
\begin{aligned}
& \left\langle q^{*}(s), q(\theta)\right\rangle=\bar{D}\left(1, \bar{\alpha}^{*}, \bar{\beta}^{*}\right)(1, \alpha, \beta)^{T} \\
& -\int_{-1}^{0} \int_{\xi=0}^{\theta} \bar{D}\left(1, \bar{\alpha}^{*}, \bar{\beta}^{*}\right) e^{-i(\xi-\theta) \omega_{0} \tau_{0}} d \eta(\theta)(1, \alpha, \beta)^{T} \\
& \quad \cdot e^{i \xi \omega_{0} \tau_{0}} d \xi=\bar{D}\left(1+\alpha \bar{\alpha}^{*}+\beta \bar{\beta}^{*}\right. \\
& \left.\quad-\int_{-1}^{0}\left(1, \bar{\alpha}^{*}, \bar{\beta}^{*}\right) \theta e^{i \theta \omega_{0} \tau_{0}} d \eta(\theta)(1, \alpha, \beta)^{T}\right)=\bar{D}(1 \\
& \left.+\alpha \bar{\alpha}^{*}+\beta \bar{\beta}^{*}-a \tau_{0} e^{-i \omega_{0} \tau_{0}}\right) .
\end{aligned}
$$

Therefore, we can choose $\bar{D}$ as

$$
\begin{aligned}
\bar{D} & =\left[1+\alpha \bar{\alpha}^{*}+\beta \bar{\beta}^{*}-a \tau_{0} e^{-i \omega_{0} \tau_{0}}\right]^{-1} \\
& =\frac{1}{\operatorname{Re}\left(\bar{D}^{-1}\right)+i \operatorname{Im}\left(\bar{D}^{-1}\right)},
\end{aligned}
$$


where $\operatorname{Re}\left(\bar{D}^{-1}\right)=1-a \tau_{0} \cos \left(\omega_{0} \tau_{0}\right)+\operatorname{Re}(\alpha) \operatorname{Re}\left(\alpha^{*}\right)+$ $\operatorname{Im}(\alpha) \operatorname{Im}\left(\alpha^{*}\right)+\operatorname{Re}(\beta) \operatorname{Re}\left(\beta^{*}\right)+\operatorname{Im}(\beta) \operatorname{Im}\left(\beta^{*}\right), \operatorname{Im}\left(\bar{D}^{-1}\right)=$ $a \tau_{0} \sin \left(\omega_{0} \tau_{0}\right)+\operatorname{Re}\left(\alpha^{*}\right) \operatorname{Im}(\alpha)-\operatorname{Re}(\alpha) \operatorname{Im}\left(\alpha^{*}\right)+\operatorname{Re}\left(\beta^{*}\right) \operatorname{Im}(\beta)-$ $\operatorname{Re}(\beta) \operatorname{Im}\left(\beta^{*}\right)$.

We can get $\left\langle q^{*}(s), \bar{q}(\theta)\right\rangle=0$.

In what follows, we will obtain the coordinates to describe the center manifold $C_{0}$ at $\tau=\tau_{0}$.

Notice that $u_{t}(\theta)=\left(x_{t}(\theta), y_{t}(\theta), z_{t}(\theta)\right)^{T}=z q(\theta)+\bar{z} \bar{q}(\theta)+$ $W(t, \theta)$; then we have

$$
\begin{aligned}
x_{t}(0)= & z+\bar{z}+W_{20}^{(1)}(0) \frac{z^{2}}{2}+W_{11}^{(1)}(0) z \bar{z} \\
& +W_{02}^{(1)}(0) \frac{\bar{z}^{2}}{2}+W_{30}^{(1)}(0) \frac{z^{3}}{6} \\
& +W_{21}^{(1)}(0) \frac{z^{2} \bar{z}}{2}+\cdots, \\
y_{t}(0)= & z \alpha+\bar{z} \bar{\alpha}+W_{20}^{(2)}(0) \frac{z^{2}}{2}+W_{11}^{(2)}(0) z \bar{z} \\
& +W_{02}^{(2)}(0) \frac{\bar{z}^{2}}{2}+W_{30}^{(2)}(0) \frac{z^{3}}{6} \\
& +W_{21}^{(2)}(0) \frac{z^{2} \bar{z}}{2}+\cdots, \\
z_{t}(0)= & z \beta+\bar{z} \bar{\beta}+W_{20}^{(3)}(0) \frac{z^{2}}{2}+W_{11}^{(3)}(0) z \bar{z} \\
& +W_{02}^{(3)}(0) \frac{\bar{z}^{2}}{2}+W_{30}^{(3)}(0) \frac{z^{3}}{6} \\
& +W_{21}^{(3)}(0) \frac{z^{2} \bar{z}}{2}+\cdots .
\end{aligned}
$$

Thus, from (29) we have

$$
\begin{aligned}
g(z, \bar{z}) & =\overline{q^{*}}(0) f_{0}(z, \bar{z}) \\
& =\bar{D} \tau_{0}\left(1, \bar{\alpha}^{*}, \bar{\beta}^{*}\right)\left(\begin{array}{c}
0 \\
-x_{t}(0) z_{t}(0) \\
x_{t}(0) y_{t}(0)
\end{array}\right) \\
& =\bar{D} \tau_{0}\left[\bar{\beta}^{*} x_{t}(0) y_{t}(0)-\bar{\alpha}^{*} x_{t}(0) z_{t}(0)\right] .
\end{aligned}
$$

Comparing the coefficients with (31), we can obtain the following formulae:

$$
\begin{aligned}
& g_{20}=2 \bar{D} \tau_{0}\left(\alpha \overline{\beta^{*}}-\beta \overline{\alpha^{*}}\right), \\
& g_{11}=2 \bar{D} \tau_{0}\left(\operatorname{Re}(\alpha) \overline{\beta^{*}}-\operatorname{Re}(\beta) \overline{\alpha^{*}}\right), \\
& g_{02}=2 \bar{D} \tau_{0}\left(\bar{\alpha} \overline{\beta^{*}}-\bar{\beta} \overline{\alpha^{*}}\right),
\end{aligned}
$$

$$
\begin{aligned}
g_{21} & =2 \bar{D} \tau_{0}\left(\overline { \beta ^ { * } } \left(\frac{1}{2} W_{20}^{(1)}(0) \bar{\alpha}+\frac{1}{2} W_{20}^{(2)}(0)\right.\right. \\
& \left.+W_{11}^{(1)}(0) \alpha+W_{11}^{(2)}(0)\right)-\overline{\alpha^{*}}\left(\frac{1}{2} W_{20}^{(1)}(0) \bar{\beta}\right. \\
& \left.\left.+\frac{1}{2} W_{20}^{(3)}(0)+W_{11}^{(1)}(0) \beta+W_{11}^{(3)}(0)\right)\right) .
\end{aligned}
$$

In order to obtain $g_{21}$, we need to compute $W_{20}(\theta)$ and $W_{11}(\theta)$. Substituting the corresponding series into (31) and comparing the coefficients, we obtain

$$
\begin{aligned}
\left(A-2 i \tau_{0} \omega_{0}\right) W_{20}(\theta) & =-H_{20}(\theta), \\
A W_{11}(\theta) & =-H_{11}(\theta) .
\end{aligned}
$$

For $\theta \in[-1,0)$, from (31), we know that

$$
\begin{aligned}
H(z, \bar{z}, \theta) & =-\bar{q}^{*}(0) f_{0} q(\theta)-q^{*}(0) \bar{f}_{0} \bar{q}(\theta) \\
& =-g q(\theta)-\bar{g} \bar{q}(\theta) .
\end{aligned}
$$

Comparing the coefficients with (31), we have

$$
\begin{aligned}
& H_{20}(\theta)=-g_{20} q(\theta)-\bar{g}_{02} \bar{q}(\theta), \\
& H_{11}(\theta)=-g_{11} q(\theta)-\bar{g}_{11} \bar{q}(\theta) .
\end{aligned}
$$

From (63), (64), and the definition of $A$, we can easily get

$$
\dot{W}_{20}(\theta)=2 i \tau_{0} \omega_{0} W_{20}(\theta)+g_{20} q(\theta)+\bar{g}_{02} \bar{q}(\theta) .
$$

Notice that $q(\theta)=(1, \alpha, \beta)^{T} e^{i \theta \omega_{0} \tau_{0}}$; hence

$$
\begin{aligned}
W_{20}(\theta)= & \frac{i g_{20}}{\tau_{0} \omega_{0}} q(0) e^{i \theta \omega_{0} \tau_{0}}+\frac{i \bar{g}_{02}}{3 \tau_{0} \omega_{0}} \bar{q}(0) e^{-i \theta \omega_{0} \tau_{0}} \\
& +E_{1} e^{2 i \theta \omega_{0} \tau_{0}}
\end{aligned}
$$

where $E_{1}=\left(E_{1}^{(1)}, E_{1}^{(2)}, E_{1}^{(3)}\right) \in R^{3}$ is a constant vector. Similarly, we can obtain

$$
\begin{aligned}
W_{11}(\theta)= & -\frac{i g_{11}}{\tau_{0} \omega_{0}} q(0) e^{i \theta \omega_{0} \tau_{0}}+\frac{i \bar{g}_{11}}{\tau_{0} \omega_{0}} \bar{q}(0) e^{-i \theta \omega_{0} \tau_{0}} \\
& +E_{2},
\end{aligned}
$$

where $E_{2}=\left(E_{2}^{(1)}, E_{2}^{(2)}, E_{2}^{(3)}\right) \in R^{3}$ is a constant vector.

In what follows, we will seek appropriate $E_{1}$ and $E_{2}$. According to the definition of $A$ and (63), we have

$$
\begin{aligned}
& \int_{-1}^{0} d \eta(\theta, 0) W_{20}(\theta)=2 i \tau_{0} \omega_{0} W_{20}(0)-H_{20}(0), \\
& \int_{-1}^{0} d \eta(\theta, 0) W_{11}(\theta)=-H_{11}(0) .
\end{aligned}
$$




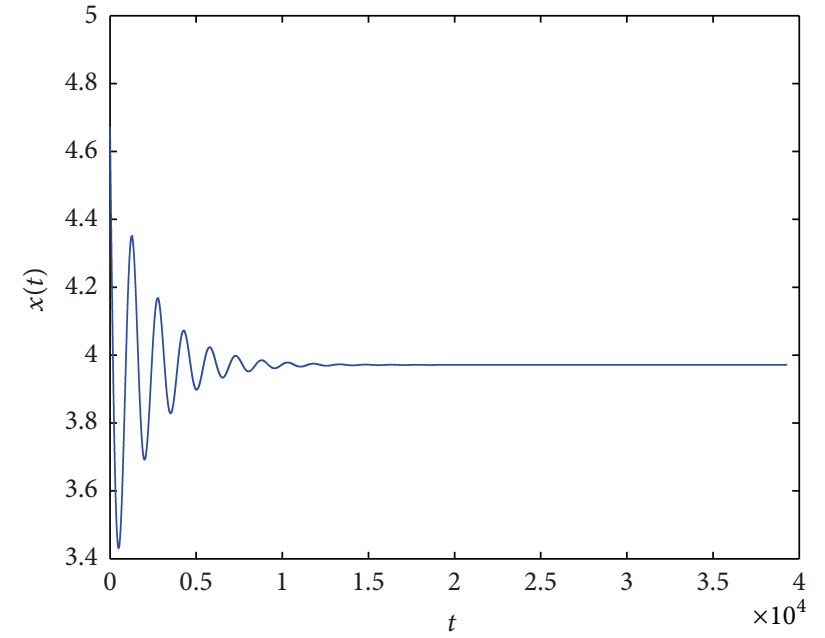

(a)

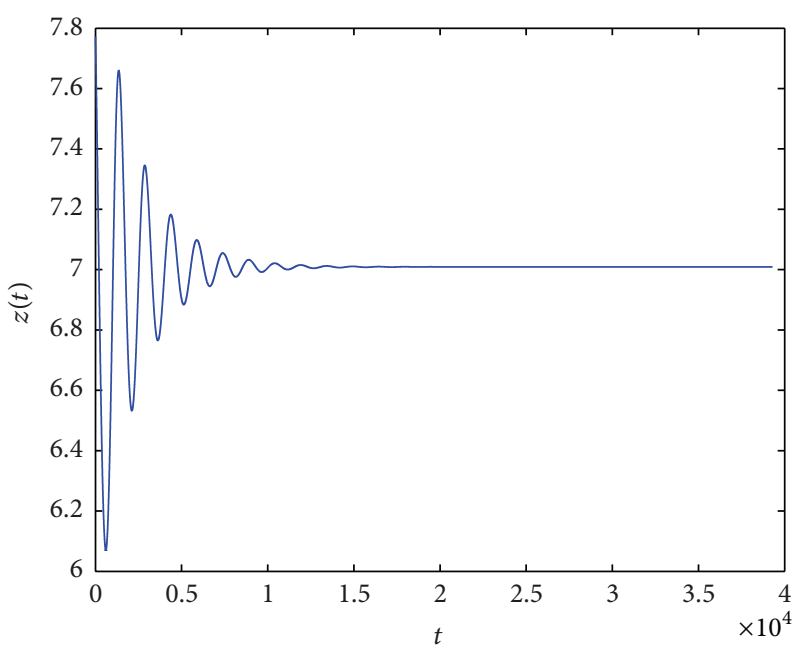

(c)

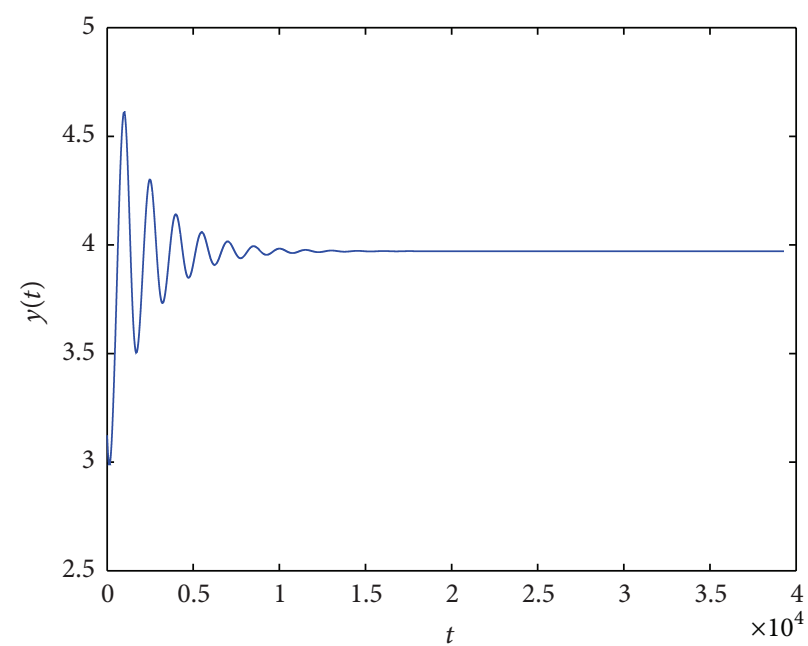

(b)

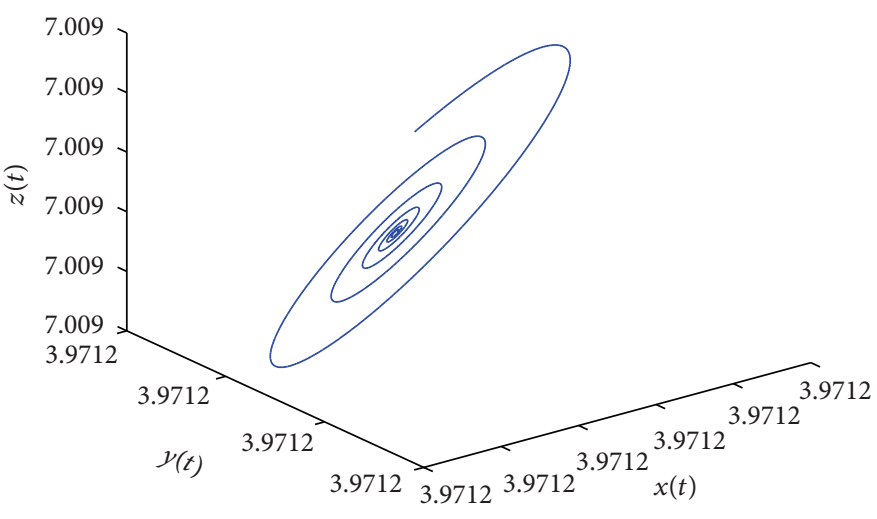

(d)

FIGURE 1: The trajectories and the phase graph of system (83) with $T=98, \varepsilon=0$, and $\tau=0.2 ; E_{2}^{*}$ is asymptotically stable.

From (31), we have

$$
\begin{aligned}
& H_{20}(0)=-g_{20} q(0)-\bar{g}_{02} \bar{q}(0)+2 \tau_{0}\left(\begin{array}{c}
0 \\
-\beta \\
\alpha
\end{array}\right), \\
& H_{11}(0)=-g_{11} q(0)-\bar{g}_{11} \bar{q}(0)+2 \tau_{0}\left(\begin{array}{c}
0 \\
-\operatorname{Re}(\beta) \\
\operatorname{Re}(\alpha)
\end{array}\right) .
\end{aligned}
$$

Substituting (67) and (71) into (69) and noticing that

$$
\begin{gathered}
\left(i \omega_{0} \tau_{0} I-\int_{-1}^{0} e^{i \omega_{0} \tau_{0} \theta} d \eta(\theta)\right) q(0)=0, \\
\left(-i \omega_{0} \tau_{0} I-\int_{-1}^{0} e^{-i \omega_{0} \tau_{0} \theta} d \eta(\theta)\right) q(0)=0,
\end{gathered}
$$

we have

$$
\left(2 i \omega_{0} \tau_{0} I-\int_{-1}^{0} e^{2 i \omega_{0} \tau_{0} \theta} d \eta(\theta)\right) E_{1}=2 \tau_{0}\left(\begin{array}{c}
0 \\
-\beta \\
\alpha
\end{array}\right)
$$

that is,

$$
\begin{aligned}
& \left(\begin{array}{ccc}
2 i \omega_{0}+a e^{-2 i \omega_{0} \tau_{0}} & -a & 0 \\
-1 & 2 i \omega_{0}+1 & x^{*} \\
-x^{*} & -x^{*} & 2 i \omega_{0}+b
\end{array}\right) E_{1} \\
& =2\left(\begin{array}{c}
0 \\
-\beta \\
\alpha
\end{array}\right) .
\end{aligned}
$$




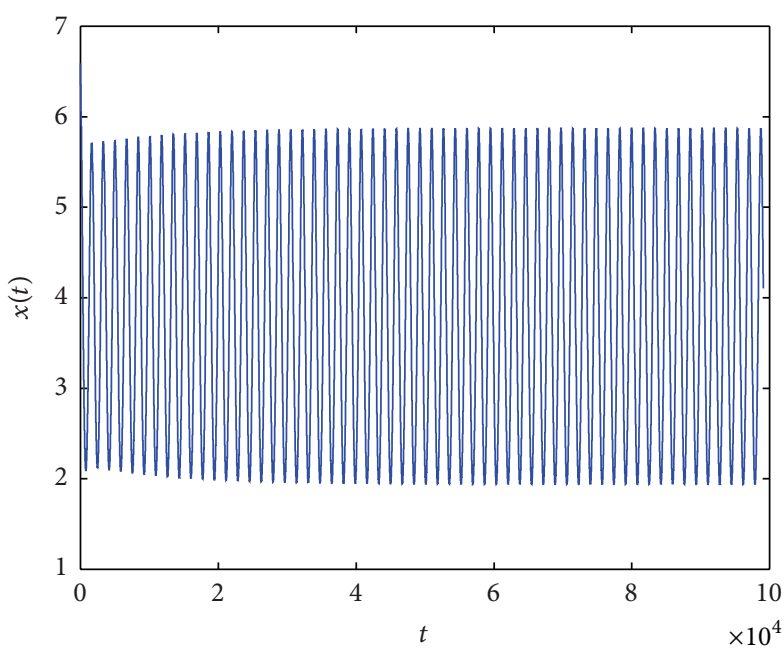

(a)

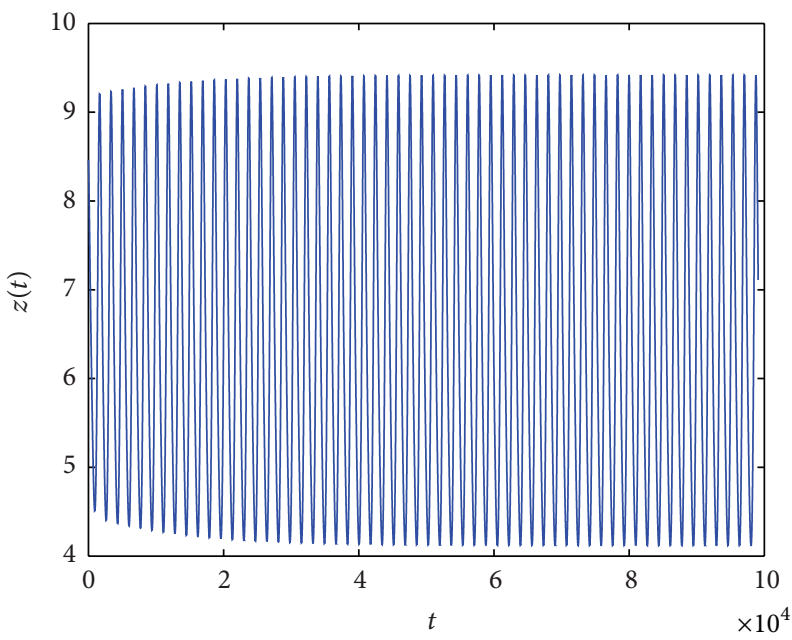

(c)

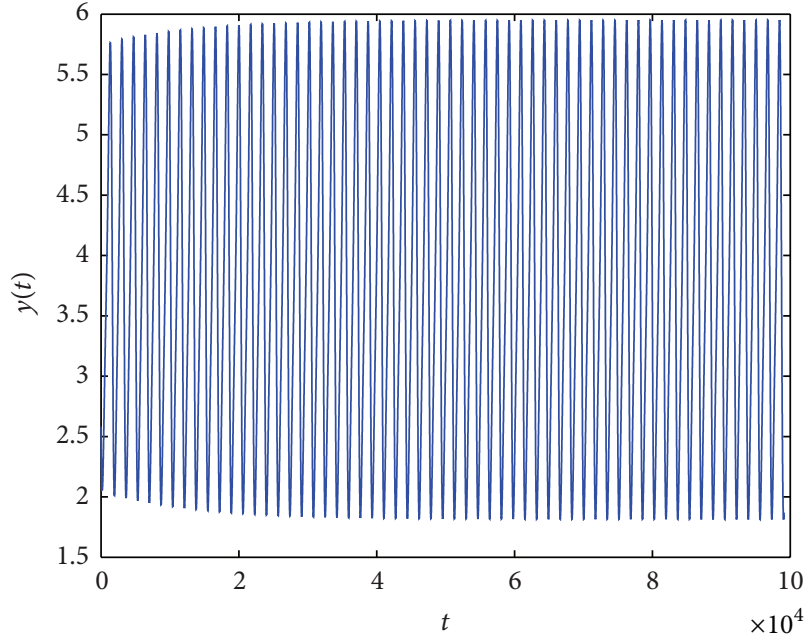

(b)

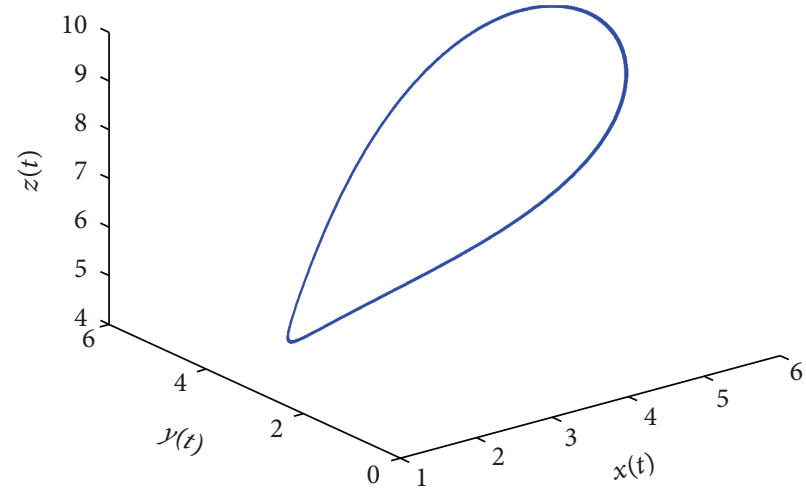

(d)

Figure 2: The trajectories and the phase graph of system (83) with $T=98, \varepsilon=0$, and $\tau=0.4$; $E_{2}^{*}$ becomes unstable, and a stable periodic solution bifurcates from equilibrium $E_{2}^{*}$.

According to Cramer's criteria, the solution of (75) is described by

$$
\begin{aligned}
E_{1} & =2\left(\begin{array}{ccc}
2 i \omega_{0}+a e^{-2 i \omega_{0} \tau_{0}} & -a & 0 \\
-1 & 2 i \omega_{0}+1 & x^{*} \\
-x^{*} & -x^{*} & 2 i \omega_{0}+b
\end{array}\right)^{-1} \\
& \cdot\left(\begin{array}{c}
0 \\
-\beta \\
\alpha
\end{array}\right)=\frac{2}{A}\left(\begin{array}{l}
a_{1} \\
a_{2} \\
a_{3}
\end{array}\right)
\end{aligned}
$$

where, $a_{1}=-a \beta\left(2 i \omega_{0}+b\right)-a x^{*} \alpha, a_{2}=-\beta\left(2 i \omega_{0}+\right.$ $\left.a e^{-2 i \omega_{0} \tau_{0}}\right)\left(2 i \omega_{0}+b\right)-x^{*} \alpha\left(2 i \omega_{0}+a e^{-2 i \omega_{0} \tau_{0}}\right), a_{3}=-\beta x^{*}\left(2 i \omega_{0}+\right.$ $\left.a e^{-2 i \omega_{0} \tau_{0}}+a\right)+\alpha\left(2 i \omega_{0}+a e^{-2 i \omega_{0} \tau_{0}}\right)\left(2 i \omega_{0}+1\right)-a \alpha$, and

$$
A=\left|\begin{array}{ccc}
2 i \omega_{0}+a e^{-2 i \omega_{0} \tau_{0}} & -a & 0 \\
-1 & 2 i \omega_{0}+1 & x^{*} \\
-x^{*} & -x^{*} & 2 i \omega_{0}+b
\end{array}\right| .
$$

Similarly, substituting (68) and (72) into (70), we have

$$
\begin{aligned}
E_{2} & =2\left(\begin{array}{ccc}
a & -a & 0 \\
-1 & 1 & x^{*} \\
-x^{*} & -x^{*} & b
\end{array}\right)^{-1}\left(\begin{array}{c}
0 \\
\operatorname{Re}(\beta) \\
\operatorname{Re}(\alpha)
\end{array}\right) \\
& =\frac{2}{B}\left(\begin{array}{c}
a b \operatorname{Re}(\beta)-a x^{*} \operatorname{Re}(\alpha) \\
a b \operatorname{Re}(\beta)-a x^{*} \operatorname{Re}(\alpha) \\
2 a x^{*} \operatorname{Re}(\beta)
\end{array}\right),
\end{aligned}
$$




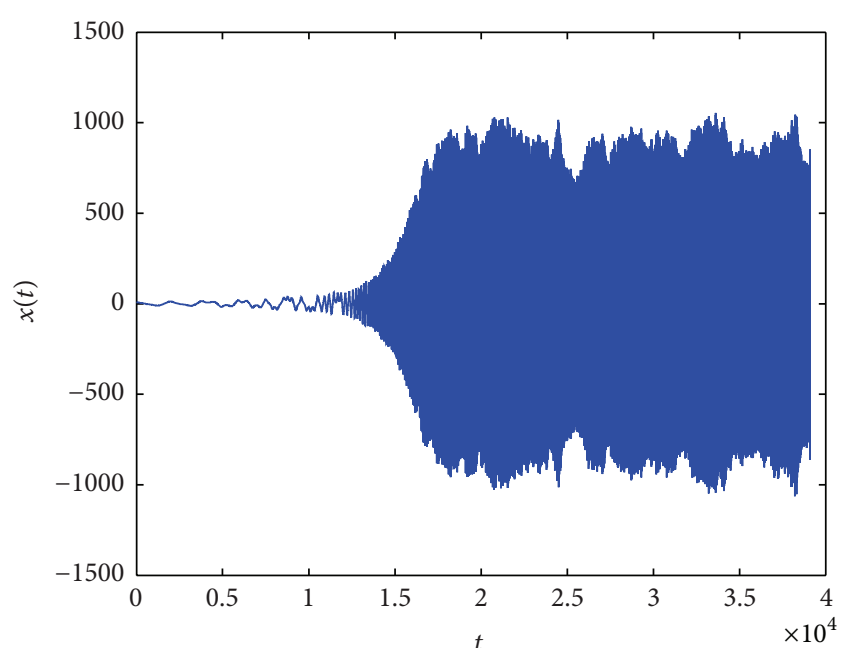

(a)

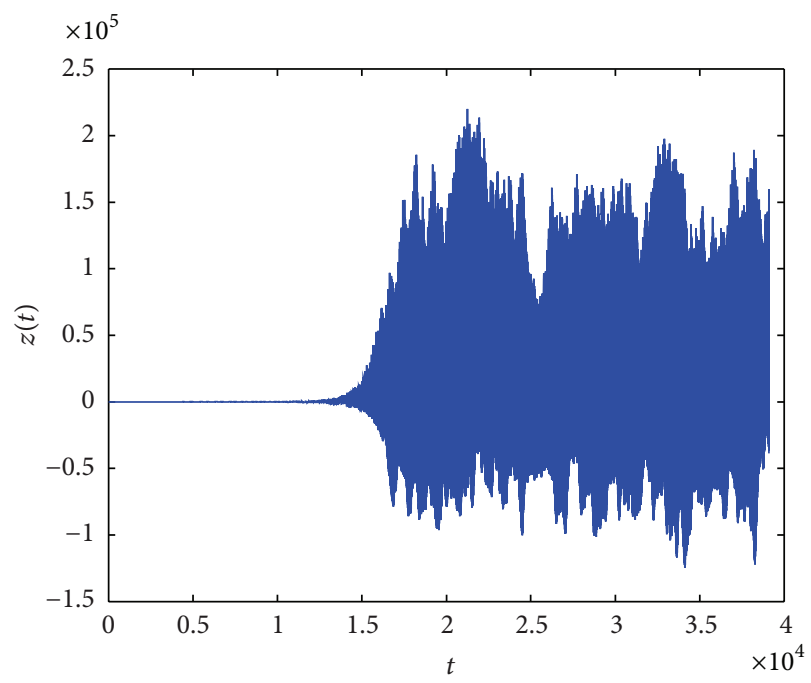

(c)

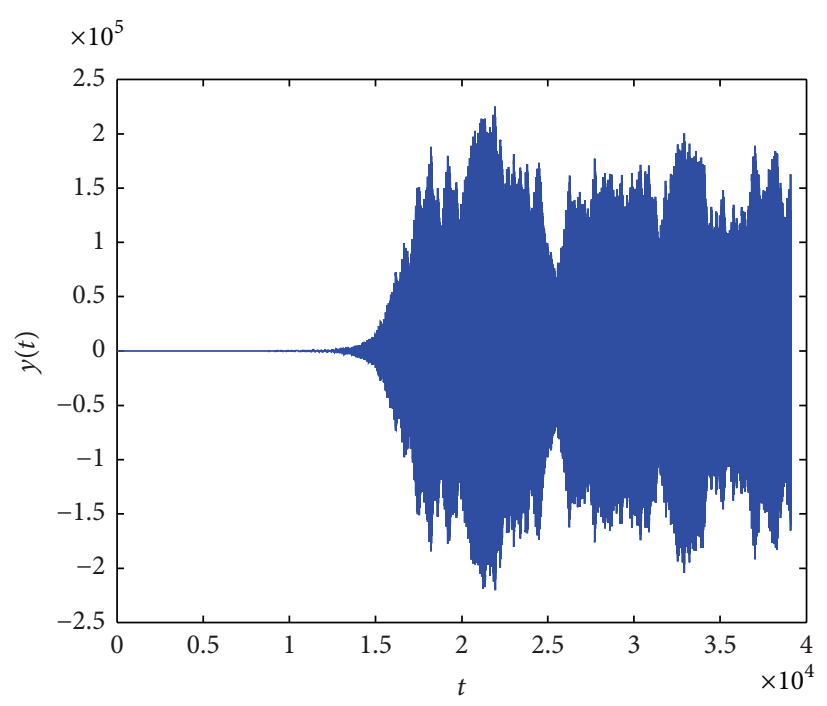

(b)

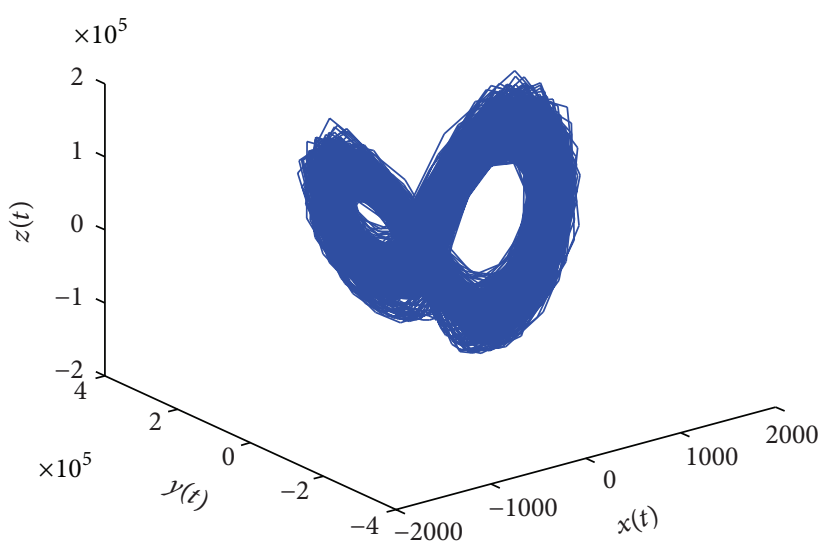

(d)

Figure 3: The trajectories and the phase graph of system (83) with $T=98, \varepsilon=0.326$, and $\tau=0.4 ; E_{2}^{*}$ is unstable, and a rank one strange attractor occurs.

where

$$
B=\left|\begin{array}{ccc}
a & -a & 0 \\
-1 & 1 & x^{*} \\
-x^{*} & -x^{*} & b
\end{array}\right| .
$$

Thus, we can compute the following values:

$$
\begin{aligned}
& \mu_{2}=-\frac{\operatorname{Re}\left\{k_{1}(0)\right\}}{\operatorname{Re}\left\{\lambda^{\prime}\left(\tau_{k}\right)\right\}}, \\
& \beta_{2}=2 \operatorname{Re}\left\{k_{1}(0)\right\},
\end{aligned}
$$

where $k_{1}(0)=\left(i / 2 \omega_{0} \tau_{0}\right)\left(g_{20} g_{11}-2\left|g_{11}\right|^{2}-(1 / 3)\left|g_{02}\right|^{2}\right)+$ $g_{21} / 2$, determining the quantities of bifurcating periodic solutions in the center manifold at the critical value $\tau_{0}$; that is, $\mu_{2}$ determines the directions of the Hopf bifurcation: if $\operatorname{Re}\left\{\lambda^{\prime}\left(\tau_{0}\right)\right\}>0, \mu_{2}>0$ (resp., $\mu_{2}<0$ ), then the Hopf bifurcation is supercritical (resp., subcritical) and the periodic solutions exist for $\tau>\tau_{0}\left(\tau<\tau_{0}\right)$. $\beta_{2}$ determines the stability of the bifurcation periodic solutions: the bifurcating periodic solutions are stable (unstable) if $\beta_{2}<0\left(\beta_{2}>0\right)$.

Furthermore, we can get the definite expressions of $\operatorname{Re}\left(k_{1}(0)\right)$ and $\operatorname{Im}\left(k_{1}(0)\right)$. From (32), we have

$$
\begin{aligned}
\Psi_{x}(x, y) & =\operatorname{Re}\left\{\overline{q^{*}}(0) \Phi(2 \operatorname{Re}(x+i y) q(0))\right\} \\
= & \operatorname{Re}\left\{\bar{D} \tau_{0}\left(1, \bar{\alpha}^{*}, \bar{\beta}^{*}\right)\right.
\end{aligned}
$$




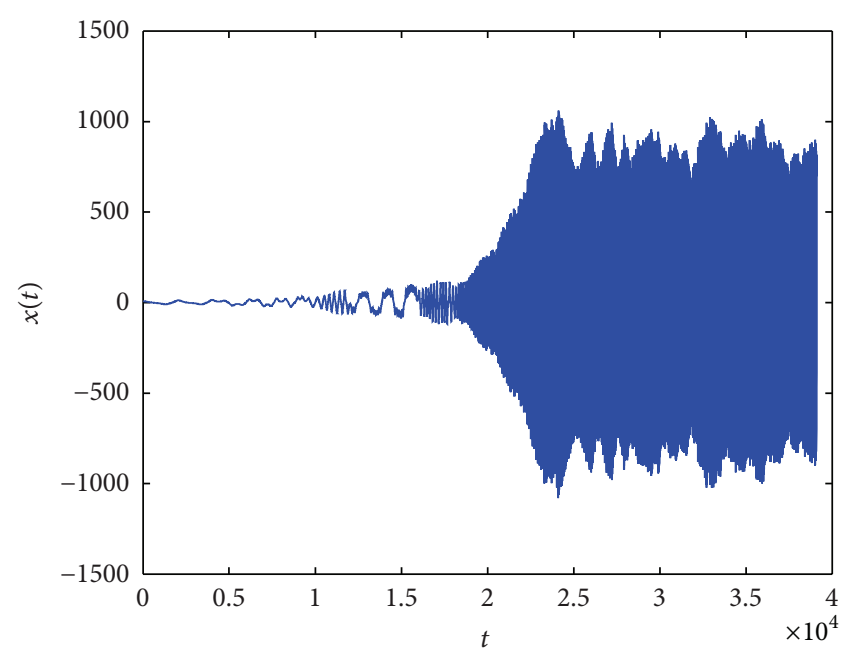

(a)

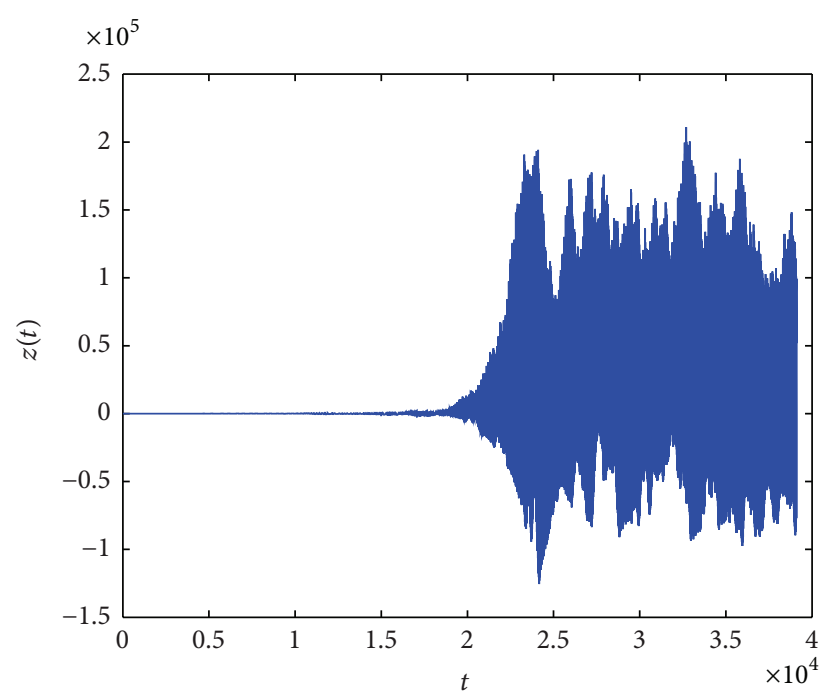

(c)

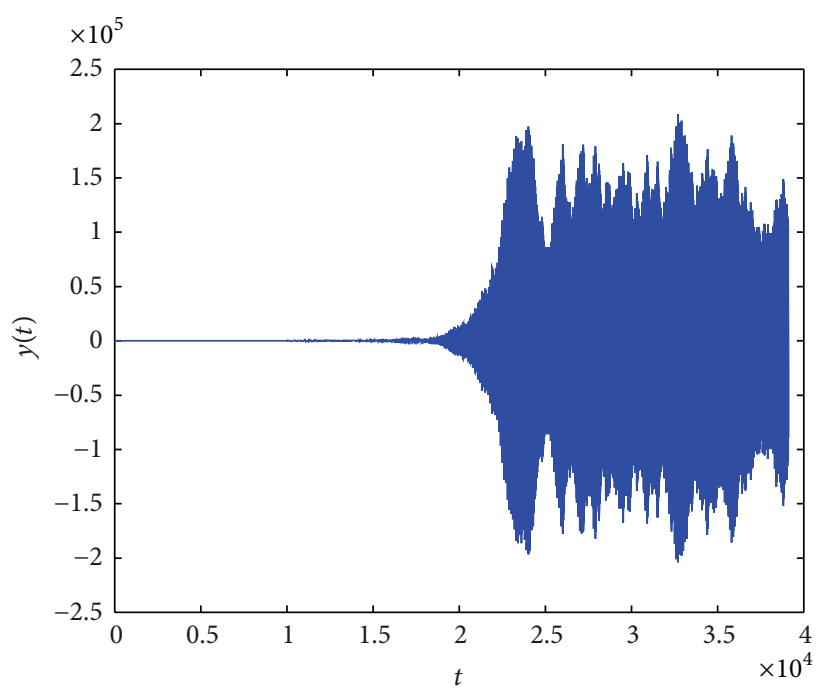

(b)

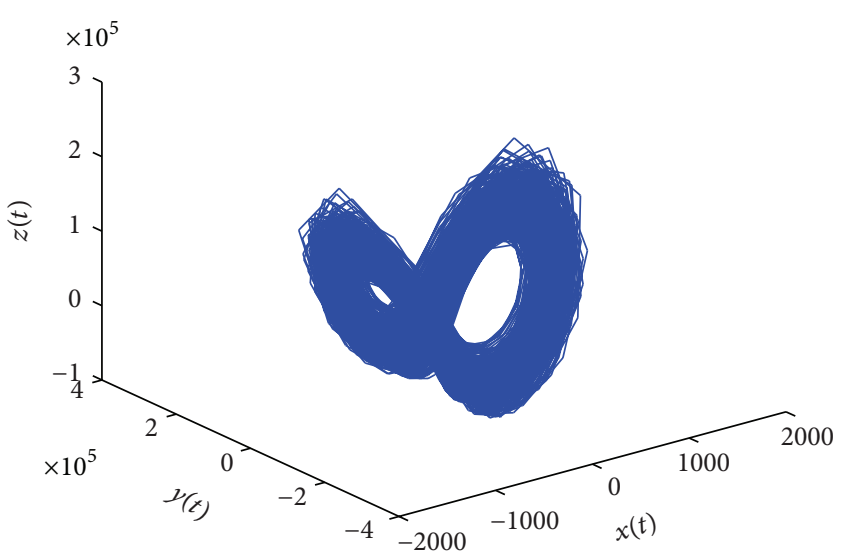

(d)

Figure 4: The trajectories and the phase graph of system (83) with $T=100, \varepsilon=0.326$, and $\tau=0.4 ; E_{2}^{*}$ is unstable, and a rank one strange attractor occurs.

$$
\begin{aligned}
& \left.\cdot\left(\begin{array}{c}
0 \\
(x+i y) \alpha+(x-i y) \bar{\alpha} \\
0
\end{array}\right)\right\} \\
& =\operatorname{Re}\left\{\bar{D} \tau_{0} \bar{\alpha}^{*}((x+i y) \alpha+(x-i y) \bar{\alpha})\right\}=2 \tau_{0}(x \\
& \cdot \operatorname{Re}(\alpha)-y \operatorname{Im}(\alpha))\left(\operatorname{Re}\left(\alpha^{*}\right) \operatorname{Re}\left(\bar{D}^{-1}\right)+\operatorname{Im}\left(\alpha^{*}\right)\right. \\
& \left.\cdot \operatorname{Im}\left(\bar{D}^{-1}\right)\right), \\
& \Psi_{y}(x, y)=\operatorname{Im}\left\{\bar{q}^{*}(0) \Phi(2 \operatorname{Re} z q(0))\right\}=2 \tau_{0}(x \\
& \cdot \operatorname{Re}(\alpha)-y \operatorname{Im}(\alpha))\left(\operatorname{Re}\left(\alpha^{*}\right) \operatorname{Im}\left(\bar{D}^{-1}\right)-\operatorname{Im}\left(\alpha^{*}\right)\right. \\
& \left.\cdot \operatorname{Re}\left(\bar{D}^{-1}\right)\right) .
\end{aligned}
$$

Let $x=\cos \theta$ and $y=\sin \theta$, then

$$
\begin{aligned}
\varphi(\theta) & =\cos \theta \Psi_{x}\left(\widehat{s}_{0}\right)+\sin \theta \Psi_{y}\left(\widehat{s}_{0}\right) \\
& =2 \tau_{0}\left[\delta_{1} \operatorname{Re}(\alpha) \cos ^{2} \theta-\delta_{2} \operatorname{Im}(\alpha) \sin ^{2} \theta\right. \\
& \left.+\left(\delta_{2} \operatorname{Re}(\alpha)-\delta_{1} \operatorname{Im}(\alpha)\right) \cos \theta \sin \theta\right],
\end{aligned}
$$

where $\delta_{1}=\left(\operatorname{Re}\left(\alpha^{*}\right) \operatorname{Re}\left(\bar{D}^{-1}\right)+\operatorname{Im}\left(\alpha^{*}\right) \operatorname{Im}\left(\bar{D}^{-1}\right)\right)$ and $\delta_{2}=$ $\left(\operatorname{Re}\left(\alpha^{*}\right) \operatorname{Im}\left(\bar{D}^{-1}\right)-\operatorname{Im}\left(\alpha^{*}\right) \operatorname{Re}\left(\bar{D}^{-1}\right)\right)$. Then $\varphi(\theta)$ is easy to verify being a Morse function from (82). According to Theorem 5, if $\operatorname{Re}\left(k_{1}(0)\right)<0$, then there exists $K_{2}$ such that when $\left|\varepsilon\left(\operatorname{Im}\left(k_{1}(0)\right) / \operatorname{Re}\left(k_{1}(0)\right)\right)\right|>K_{2}$, we can get an observable rank one chaos. 


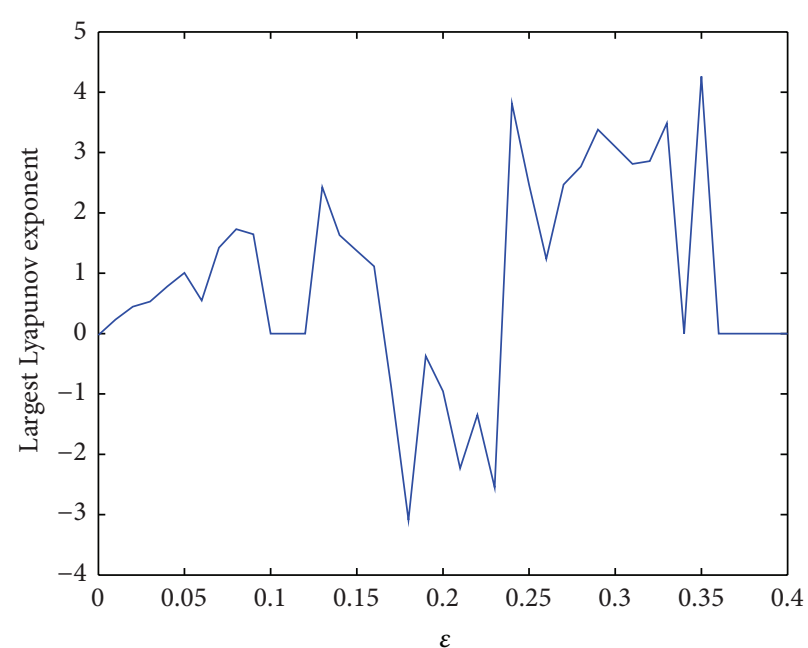

(a)

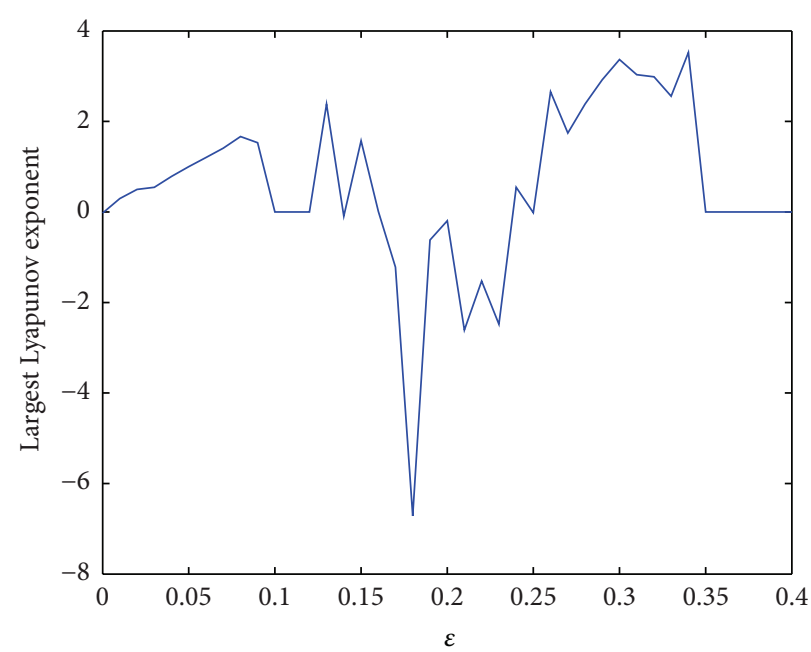

(b)

FIGURE 5: Largest Lyapunov exponent $\lambda$ versus $\varepsilon$ for system (83) with $T=98$ (a), $T=100$ (b), $\tau=0.4$, and $\varepsilon$ varying from 0 to 0.4 .

\section{Numerical Simulations}

Now we move to the parameters for which rank one attractors are observable, so we consider the following system:

$$
\begin{aligned}
& \dot{x}(t)=1.9(y(t)-x(t-\tau)), \\
& \dot{y}(t)=8.009 x(t)-x(t) z(t)-y(t)+\varepsilon P_{T} y(t), \\
& \dot{z}(t)=x(t) y(t)-2.25 z(t) .
\end{aligned}
$$

System (83) has a positive equilibrium $E_{2}^{*}(3.9712,3.9712$, $7.0090)$. It is not difficult to get the following linear system of (83) at $E_{2}^{*}$ without the disturbed term $\varepsilon P_{T} y(t)$ :

$$
\begin{aligned}
& \dot{u}_{1}(t)=-1.9 x(t-\tau)+1.9 y(t), \\
& \dot{u}_{2}(t)=x(t)-y(t)-3.9712 z(t), \\
& \dot{u}_{3}(t)=3.9712 x(t)-3.9712 y(t)-2.25 z(t) .
\end{aligned}
$$

It follows that the characteristic equation of system (84) is

$$
\begin{aligned}
\lambda^{3}+ & 3.2500 \lambda^{2}+16.1203 \lambda+25.6885 \\
& +1.9 e^{-\lambda \tau}\left(\lambda^{2}+3.2500 \lambda+18.0203\right)=0
\end{aligned}
$$

From the discussion of Section 4 , we have $\omega_{0} \doteq 3.9355$, $\tau_{0} \doteq 0.375, \operatorname{Re}\left\{\lambda^{\prime}(0)\right\} \doteq 0.1975, \operatorname{Re}\left\{k_{1}(0)\right\} \doteq-0.0027$, $\mu_{2} \doteq 0.0139$, and $\beta_{2} \doteq-0.0055$, so we know that the equilibrium $E_{2}^{*}$ is asymptotically stable when $\varepsilon=0$ and $\tau \epsilon$ $[0,0.375)$ (see Figure 1). Further, we know that the bifurcation is supercritical and the stable periodic solution emerges from the equilibrium (see Figure 2).

We also get $\left|\operatorname{Im}\left(k_{1}(0)\right) / \operatorname{Re}\left(k_{1}(0)\right)\right| \doteq 70.6999$. Choose $T$ in interval $(98,100)$, which is quite "large" to afford a long relaxation period between consecutive kicks of the external force, and choose $\varepsilon$ in $(0,0.4)$. In Figure 3 , we present a rank one strange attractor at $(\tau, \varepsilon, T)=(0.4,0.326,98)$. In Figure 4 ,

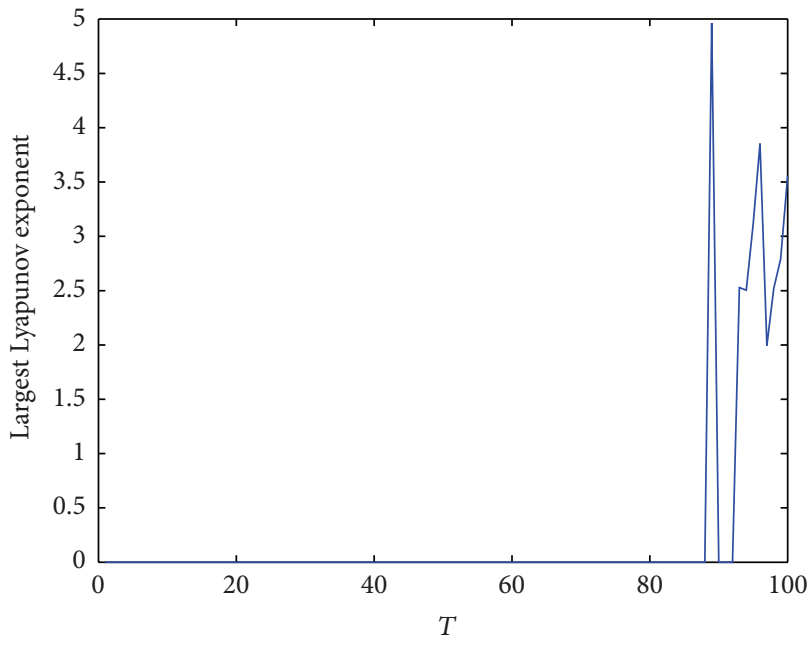

Figure 6: Largest Lyapunov exponent $\lambda$ versus $T$ for system (83) with $\varepsilon=0.326, \tau=0.4$, and $T$ varying from 0 to 120 .

a rank one strange attractor at $(\tau, \varepsilon, T)=(0.4,0.326,100)$ is shown. In Figure 5, largest Lyapunov exponent $\lambda$ versus $\varepsilon$ for system (83) with $T=98$ and $T=100$ is given. We can see that there exists a positive measure set $\Delta \subset\left(0.4^{-1}, \infty\right)$ for $T$, such that, for $T \in \Delta$, the time- $T$ map $F_{T}$ of system (83) has a strange attractor. Largest Lyapunov exponent $\lambda$ versus $T$ for system (83) with $\tau=0.4, \varepsilon=0.326$, and $T$ varying from 0 to 120 is given in Figure 6 . The results of our numerical simulations are in perfect agreement with the predictions of the rank one theory in Section 3.

\section{Conflict of Interests}

The authors declare that there is no conflict of interests regarding the publication of this paper. 


\section{Acknowledgment}

This research is supported by the National Natural Science Foundation of China (nos. 11061016 and 11461036).

\section{References}

[1] J. G. Sinai, "Gibbs measures in ergodic theory," Russian Mathematical Surveys, vol. 27, pp. 21-69, 1972.

[2] D. Ruelle, "A measure associated with Axiom A attractors," The American Journal of Mathematics, vol. 98, no. 3, pp. 619-654, 1976.

[3] R. Bowen, Equilibrium States and the Ergodic Theory of Anosov Diffeomorphisms, Springer, Berlin, Germany, 1975.

[4] J.-P. Eckmann and D. Ruelle, "Ergodic theory of chaos and strange attractors," Reviews of Modern Physics, vol. 57, no. 3, pp. 617-656, 1985.

[5] C. Bonatti, L. Diaz, and M. Viana, Dynamics Beyond Uniform Hyperbolicity, Encyclopaedia of Mathematics, 2005.

[6] E. Mihailescu, "Local geometry and dynamical behavior on folded basic sets," Journal of Statistical Physics, vol. 142, no. 1, pp. 154-167, 2011.

[7] Q. Wang and L.-S. Young, "Strange attractors with one direction of instability," Communications in Mathematical Physics, vol. 218, no. 1, pp. 1-97, 2001.

[8] Q. Wang and L.-S. Young, "Toward a theory of rank one attractors," Annals of Mathematics, vol. 167, no. 2, pp. 349-480, 2008.

[9] F. Chen and M. Han, "Rank one chaos in a class of planar systems with heteroclinic cycle," Chaos, vol. 19, no. 4, Article ID 043122, 2009.

[10] H. Fang, "Synchronization of two rank-one chaotic systems without and with delay via linear delayed feedback control," Journal of Applied Mathematics, vol. 2012, Article ID 325131, 15 pages, 2012.

[11] Q. Wang and L.-S. Young, "Dynamical profile of a class of rankone attractors," Ergodic Theory and Dynamical Systems, vol. 33, no. 4, pp. 1221-1264, 2013.

[12] E. N. Lorenz, "Deterministic nonperiodic flow," Journal of the Atmospheric Sciences, vol. 20, no. 2, pp. 130-141, 1963.

[13] J. L. Kaplan and J. A. Yorke, "Preturbulence: a regime observed in a fluid flow model of Lorenz," Communications in Mathematical Physics, vol. 67, no. 2, pp. 93-108, 1979.

[14] J.-H. Lü, G.-R. Chen, and Y.-G. Yu, "Asymptotic analysis of a modified Lorenz system," Chinese Physics Letters, vol. 19, no. 9, pp. 1260-1263, 2002.

[15] P. Yu and F. Xu, "A common phenomenon in chaotic systems linked by time delay," International Journal of Bifurcation and Chaos in Applied Sciences and Engineering, vol. 16, no. 12, pp. 3727-3736, 2006.

[16] Q. Wang and A. Oksasoglu, "Rank one chaos: theory and applications," International Journal of Bifurcation and Chaos in Applied Sciences and Engineering, vol. 18, no. 5, pp. 1261-1319, 2008.

[17] L.-S. Young, "What are SRB measures, and which dynamical systems have them?" Journal of Statistical Physics, vol. 108, no. 5-6, pp. 733-754, 2002.

[18] Q. Wang and L.-S. Young, "Strange attractors in periodicallykicked limit cycles and Hopf bifurcations," Communications in Mathematical Physics, vol. 240, no. 3, pp. 509-529, 2003.
[19] J. Guckenheimer and P. Holmes, Nonlinear Oscillations, Dynamical Systems and Bifurcations of Vector Fields, Applied Mathematical Sciences, Springer, New York, NY, USA, 5th edition, 1997.

[20] B. Hassard, D. Kazarinoff, and Y. Wan, Theory and Applications of Hopf Bifurcation, Cambridge University Press, Cambridge, UK, 1981.

[21] S. Ruan and J. J. Wei, "On the zeros of transcendental functions with applications to stability of delay differential equations with two delays," Dynamics of Continuous, Discrete and Impulsive Systems. Series A. Mathematical Analysis, vol. 10, no. 6, pp. 863874, 2003.

[22] S. Chow and J. K. Hale, Methods of Bifurcation Theory, Springer, Berlin, Germany, 1981. 


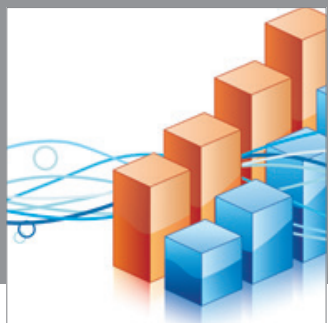

Advances in

Operations Research

mansans

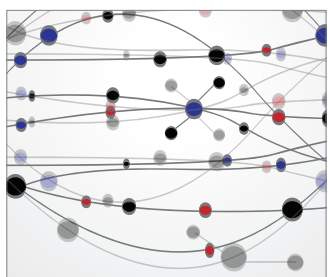

The Scientific World Journal
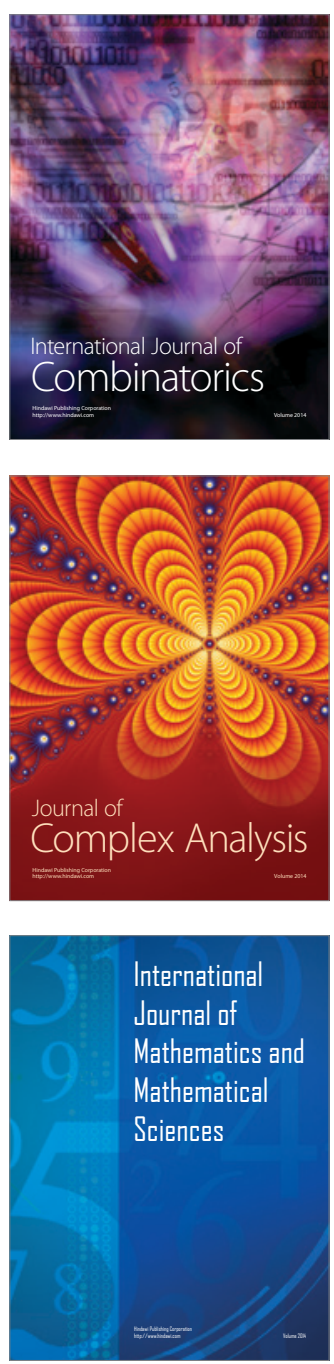
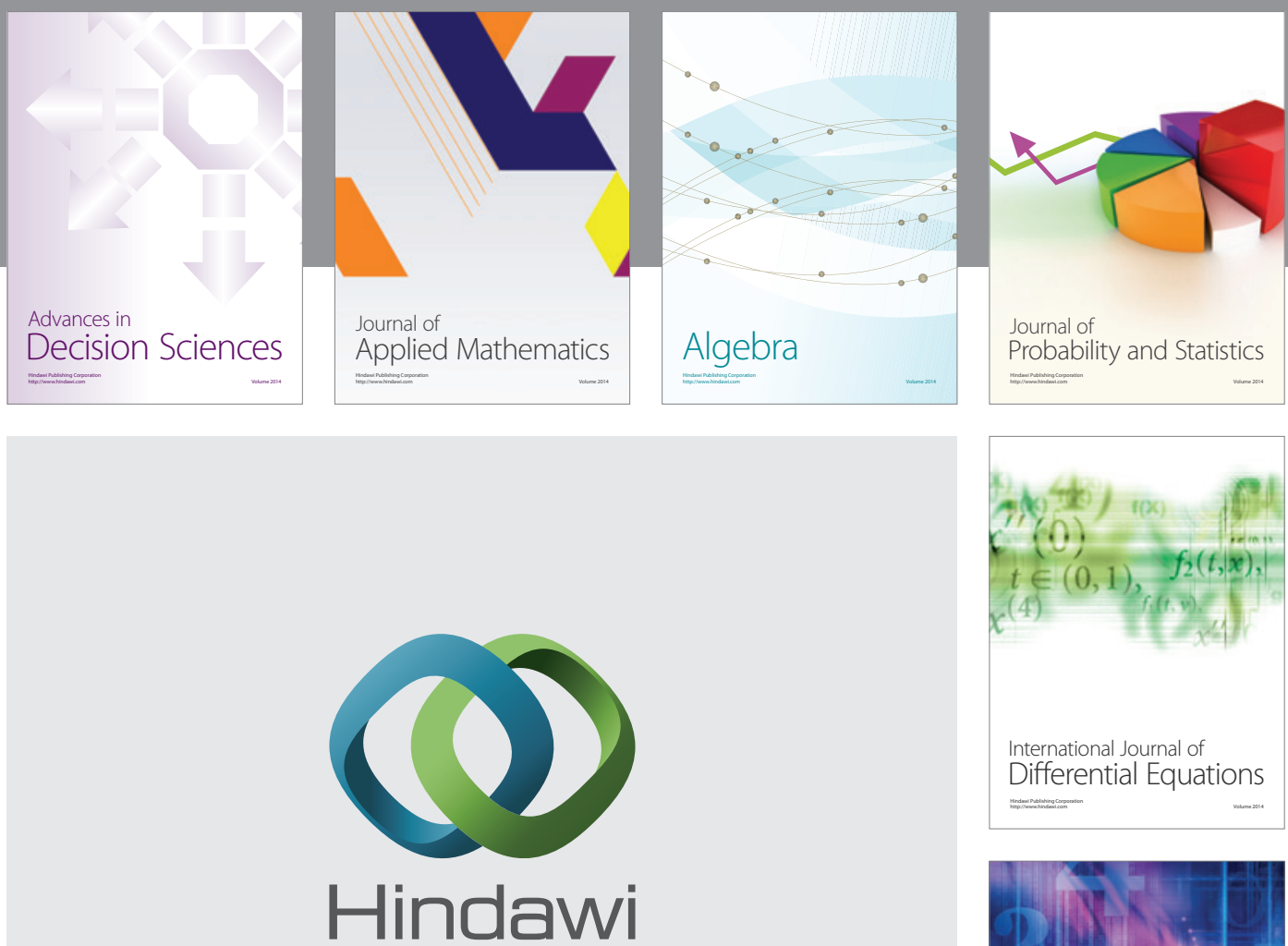

Submit your manuscripts at http://www.hindawi.com
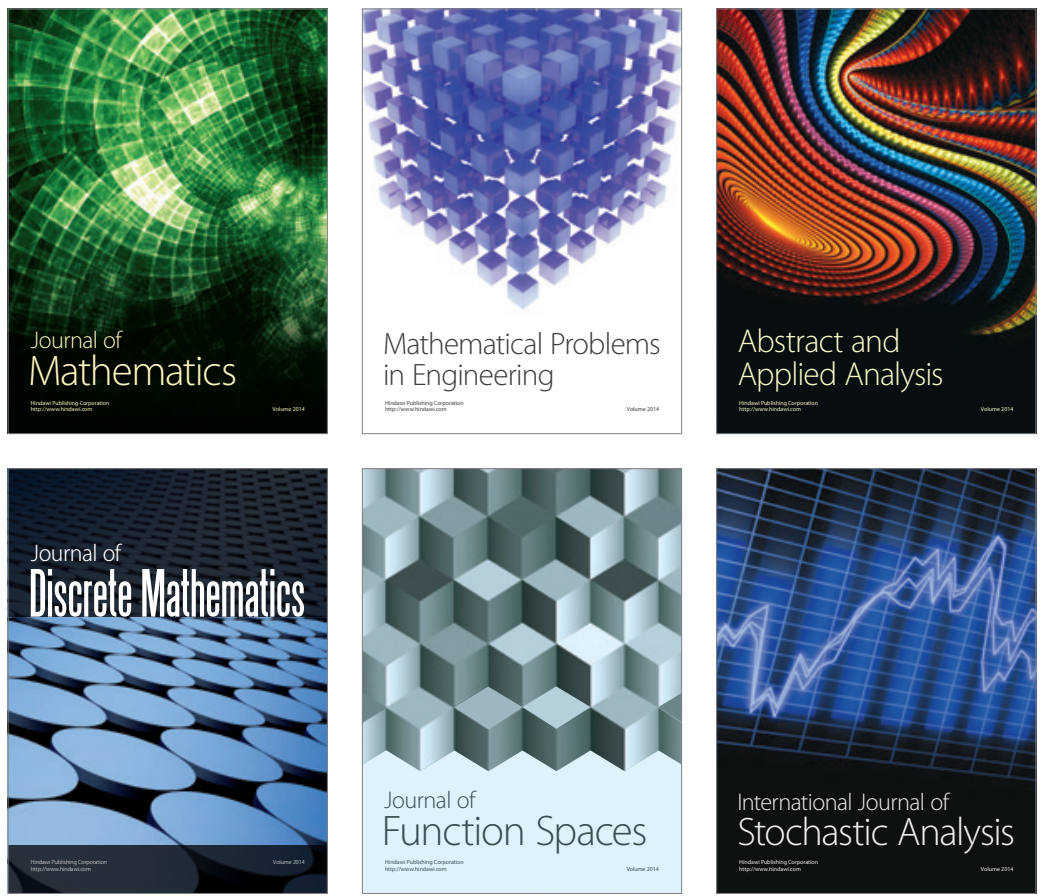

Journal of

Function Spaces

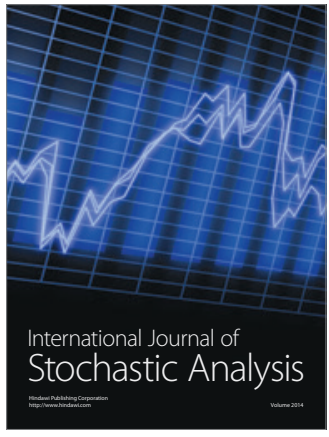

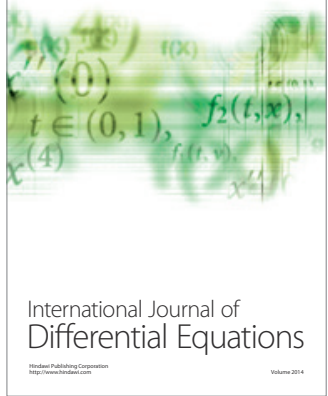
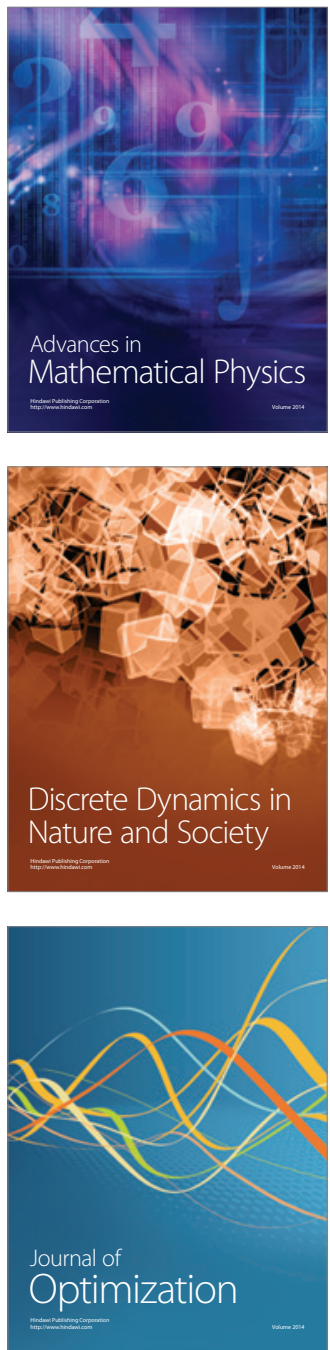